\title{
Finite Iterative Algorithm for Solving a Complex of Conjugate and Transpose Matrix Equation
}

\author{
Mohamed A. Ramadan, ${ }^{1}$ Talaat S. El-Danaf, ${ }^{1}$ and Ahmed M. E. Bayoumi ${ }^{2}$ \\ ${ }^{1}$ Department of Mathematics, Faculty of Science, Menoufia University, Shebeen El-Koom, Egypt \\ ${ }^{2}$ Department of Mathematics, Faculty of Education, Ain Shams University, Cairo, Egypt
}

Correspondence should be addressed to Mohamed A. Ramadan; mramadan@eun.eg

Received 4 August 2012; Accepted 4 November 2012

Academic Editor: Franck Petit

Copyright (C) 2013 Mohamed A. Ramadan et al. This is an open access article distributed under the Creative Commons Attribution License, which permits unrestricted use, distribution, and reproduction in any medium, provided the original work is properly cited.

We consider an iterative algorithm for solving a complex matrix equation with conjugate and transpose of two unknowns of the form: $A_{1} V B_{1}+C_{1} W D_{1}+A_{2} \bar{V} B_{2}+C_{2} \bar{W} D_{2}+A_{3} V^{H} B_{3}+C_{3} W^{H} D_{3}+A_{4} V^{T} B_{4}+C_{4} W^{T} D_{4}=E$. With the iterative algorithm, the existence of a solution of this matrix equation can be determined automatically. When this matrix equation is consistent, for any initial matrices $V_{1}, W_{1}$ the solutions can be obtained by iterative algorithm within finite iterative steps in the absence of round-off errors. Some lemmas and theorems are stated and proved where the iterative solutions are obtained. A numerical example is given to illustrate the effectiveness of the proposed method and to support the theoretical results of this paper.

\section{Introduction}

Consider the complex matrix equation:

$$
\begin{aligned}
A_{1} V B_{1} & +C_{1} W D_{1}+A_{2} \bar{V} B_{2}+C_{2} \bar{W} D_{2} \\
& +A_{3} V^{H} B_{3}+C_{3} W^{H} D_{3}+A_{4} V^{T} B_{4}+C_{4} W^{T} D_{4}=E,
\end{aligned}
$$

where $A_{1}, A_{2}, C_{1}, C_{2} \in \mathbb{C}^{m \times r}, B_{1}, B_{2}, D_{1}, D_{2} \in \mathbb{C}^{s \times n}$, $A_{3}, A_{4}, C_{3}, C_{4} \in \mathbb{C}^{m \times s}, E \in \mathbb{C}^{m \times n}$ and $B_{3}, B_{4}, D_{3}, D_{4} \in$ $\mathbb{C}^{r \times n}$ are given matrices, while $V, W \in \mathbb{C}^{r \times s}$ are matrices to be determined. In the field of linear algebra, iterative algorithms for solving matrix equations have received much attention. Based on the iterative solutions of matrix equations, Ding and Chen presented the hierarchical gradient iterative algorithms for general matrix equations $[1,2]$ and hierarchical least squares iterative algorithms for generalized coupled Sylvester matrix equations and general coupled matrix equations [3, $4]$. The hierarchical gradient iterative algorithms $[1,2]$ and hierarchical least squares iterative algorithms $[1,4,5]$ for solving general (coupled) matrix equations are innovational and computationally efficient numerical ones and were proposed based on the hierarchical identification principle $[3,6]$ which regards the unknown matrix as the system parameter matrix to be identified. Iterative algorithms were proposed for continuous and discrete Lyapunov matrix equations by applying the hierarchical identification principle [7]. Recently, the idea of the hierarchical identification was also utilized to solve the so-called extended Sylvester-conjugate matrix equations in [8]. From an optimization point of view, a gradient-based iteration was constructed in [9] to solve the general coupled matrix equation. A significant feature of the method in [9] is that a necessary and sufficient condition guaranteeing the convergence of the algorithm can be explicitly obtained.

Some complex matrix equations have attracted attention from many researchers since it was shown in [10] that the consistence of the matrix equation $A X-\bar{X} B=C$ can be characterized by the consimilarity [11-13] of two partitioned matrices related to the coefficient matrices $A, B$, and $C$. By consimilarity Jordan decomposition, explicit solutions were obtained in $[10,14]$. Some explicit expressions of the solution to the matrix equation $A X-\bar{X} B=C$ were established in [15], and it was shown that this matrix equation has a unique solution if and only if $A \bar{A}$ and $B \bar{B}$ hav no common eigenvalues. Research on solving linear matrix equations has been actively engaged in for many years. For example, Navarra et al. 
studied a representation of the general common solution of the matrix equations $A_{1} X B_{1}=C_{1}$ and $A_{2} X B_{2}=C_{2}$ [16]; Van der Woude obtained the existence of a common solution $X$ for the matrix equations $A_{i} X B_{j}=C_{i j}$ [17]; Bhimasankaram considered the linear matrix equations $A X=B, C X=D$, and $E X F=G$ [18]. Mitra has provided conditions for the existence of a solution and a representation of the general common solution of the matrix equations $A X=C$ and $X B=$ $D$ and the matrix equations $A_{1} X B_{1}=C_{1}$ and $A_{2} X B_{2}=C_{2}$ $[19,20]$. Ramadan et al. [21] introduced a complete, general, and explicit solution to the Yakubovich matrix equation $V$ $A V F=B W$, and the matrix equation $(A X B, G X H)=(C, D)$ has some important results that have been developed. In [22], necessary and sufficient conditions for its solvability and the expression of the solution were derived by means of generalized inverse. Moreover, in [22] the least-squares solution was also obtained by using the generalized singular value decomposition. While in [23], when this matrix equation is consistent, the minimum-norm solution was given by the use of the canonical correlation decomposition. In [24], based on the projection theorem in Hilbert space, an analytical expression of the least-squares solution was given for the matrix equation $(A X B, G X H)=(C, D)$ by making use of the generalized singular value decomposition and the canonical correlation decomposition. In [25], by using the matrix rank method a necessary and sufficient condition was derived for the matrix equations $A X_{1} B=C$ and $G X_{2} H=D$ to have a common least square solution. In the aforementioned methods, the coefficient matrices of the considered equations are required to be firstly transformed into some canonical forms. Recently, an iterative algorithm has presented in [26] to solve the matrix equation $(A X B, G X H)=(C, D)$. Different from the above mentioned methods, this algorithm can be implemented by initial coefficient matrices and can provide a solution within finite iteration steps for any initial values.

Very recently, in [27] a new operator of conjugate product for complex polynomial matrices is proposed. It is shown that an arbitrary complex polynomial matrix can be converted into the so-called Smith normal form by elementary transformations in the framework of conjugate product. Meanwhile, the conjugate product and the Sylvester-conjugate sum are also proposed in [28]. Based on the important properties of the above new operators, a unified approach to solve a general class of Sylvester-polynomial-conjugate matrix equations is given. The complete solution of the Sylvester-polynomialconjugate matrix equation is obtained. In [29] by using a real inner product in complex matrix spaces, a solution can be obtained within finite iterative steps for any initial values in the absence of round-off errors. In [30] iterative solutions to a class of complex matrix equations are given by applying the hierarchical identification principle.

This paper is organized as follows. First, in Section 2, we introduce some notations, a lemma, and a theorem that will be needed to develop this work. In Section 3, we propose iterative methods to obtain numerical solution to the complex matrix equation with conjugate and transpose of two unknowns of the form: $A_{1} V B_{1}+C_{1} W D_{1}+A_{2} \bar{V} B_{2}+C_{2} \bar{W} D_{2}+$ $A_{3} V^{H} B_{3}+C_{3} W^{H} D_{3}+A_{4} V^{T} B_{4}+C_{4} W^{T} D_{4}=E$ using iterative method. In Section 4, numerical example is given to explore the simplicity and the neatness of the presented methods.

\section{Preliminaries}

The following notations, definitions, lemmas, and theorems will be used to develop the proposed work. We use $A^{T}, \bar{A}, A^{H}, \operatorname{tr}(A)$, and $\|A\|$ to denote the transpose, conjugate, conjugate transpose, the trace, and the Frobenius norm of a matrix $A$, respectively. We denote the set of all $m \times n$ complex matrices by $\mathbb{C}^{m \times n}$, and $\operatorname{Re}(a)$ denote the real part of number $a$.

Definition 1 (inner product [31]). A real inner product space is a vector space $V$ over the real field $\mathbb{R}$ together with an inner product. That is, with a map

$$
\langle\cdot, \cdot\rangle: V \times V \longrightarrow \mathbb{R}
$$

Satisfying the following three axioms for all vectors $x, y, z \in$ $V$ and all scalars $a \in \mathbb{R}$ :

(1) symmetry: $\langle x, y\rangle=\langle y, x\rangle$

(2) linearity in the first argument:

$$
\begin{gathered}
\langle a x, y\rangle=a\langle x, y\rangle, \\
\langle x+y, z\rangle=\langle x, z\rangle+\langle y, z\rangle
\end{gathered}
$$

(3) positive definiteness: $\langle x, x\rangle>0$ for all $x \neq 0$, 0 .

two vectors $u, v \in V$ are said to be orthogonal if $\langle u, v\rangle=$

The following theorem defines a real inner product on space $\mathbb{C}^{m \times n}$ over the field $\mathbb{R}$.

Theorem 2 (see [32]). In the space $\mathbb{C}^{m \times n}$ over the field $\mathbb{R}$, an inner product can be defined as

$$
\langle A, B\rangle=\operatorname{Re}\left[\operatorname{tr}\left(A^{H} B\right)\right] .
$$

\section{The Main Result}

In this section, we propose an iterative solution to a complex matrix equation with conjugate and transpose of two unknowns:

$$
\begin{gathered}
A_{1} V B_{1}+C_{1} W D_{1}+A_{2} \bar{V} B_{2}+C_{2} \bar{W} D_{2}+A_{3} V^{H} B_{3} \\
+C_{3} W^{H} D_{3}+A_{4} V^{T} B_{4}+C_{4} W^{T} D_{4}=E
\end{gathered}
$$

defined in (1) where $A_{1}, A_{2}, C_{1}, C_{2} \in \mathbb{C}^{m \times r}, B_{1}, B_{2}, D_{1}$, $D_{2} \in \mathbb{C}^{s \times n}, A_{3}, A_{4}, C_{3}, C_{4} \in \mathbb{C}^{m \times s}, E \in \mathbb{C}^{m \times n}$ and $B_{3}, B_{4}$, $D_{3}, D_{4} \in \mathbb{C}^{r \times n}$ are given matrices, while $V, W \in \mathbb{C}^{r \times s}$ are matrices to be determined.

The following finite iterative algorithm is presented to solve it.

Algorithm 3. (1) Input $A_{1}, A_{2}, C_{1}, C_{2}, B_{1}, B_{2}, D_{1}, D_{2}, A_{3}, A_{4}$, $C_{3}, C_{4}, B_{3}, B_{4}, D_{3}, D_{4}, E$;

(2) Chosen arbitrary matrices $V_{1}, W_{1} \in \mathbb{C}^{r \times s}$; 
(3) Set

$$
\begin{gathered}
R_{1}=E-A_{1} V_{1} B_{1}-C_{1} W_{1} D_{1}-A_{2} \overline{V_{1}} B_{2} \\
-C_{2} \bar{W}_{1} D_{2}-A_{3} V_{1}^{H} B_{3}-C_{3} W_{1}^{H} D_{3} \\
-A_{4} V_{1}^{T} B_{4}-C_{4} W_{1}^{T} D_{4}, \\
P_{1}=A_{1}^{H} R_{1} B_{1}^{H}+\bar{A}_{2}^{H} \overline{R_{1}} \bar{B}_{2}^{H}+B_{3} R_{1}^{H} A_{3}+\overline{B_{4}} R_{1}^{T} \overline{A_{4}}, \\
Q_{1}=C_{1}^{H} R_{1} D_{1}^{H}+\bar{C}_{2}^{H} \overline{R_{1}} \bar{D}_{2}^{H}+D_{3} R_{1}^{H} C_{3}+\overline{D_{4}} R_{1}^{T} \overline{C_{4}}, \\
k=: 1 ;
\end{gathered}
$$

(4) If $R_{k}=0$, then stop; else go to Step 5;

(5) Set

$$
\begin{aligned}
V_{k+1}=V_{k}+\frac{\left\|R_{k}\right\|^{2}}{\left\|P_{k}\right\|^{2}+\left\|Q_{k}\right\|^{2}} P_{k}, \\
W_{k+1}=W_{k}+\frac{\left\|R_{k}\right\|^{2}}{\left\|P_{k}\right\|^{2}+\left\|Q_{k}\right\|^{2}} Q_{k}, \\
R_{k+1}=E-A_{1} V_{k+1} B_{1}-C_{1} W_{k+1} D_{1}-A_{2} \overline{V_{k+1}} B_{2} \\
-C_{2} \bar{W}_{k+1} D_{2}-A_{3} V_{k+1}^{H} B_{3}-C_{3} W_{k+1}^{H} D_{3} \\
-A_{4} V_{k+1}^{T} B_{4}-C_{4} W_{k+1}^{T} D_{4}, \\
P_{k+1}=A_{1}^{H} R_{k+1} B_{1}^{H}+\bar{A}_{2}^{H} \overline{R_{k+1}} \bar{B}_{2}^{H}+B_{3} R_{k+1}^{H} A_{3} \\
+\bar{B}_{4} R_{k+1}^{T} \overline{A_{4}}+\frac{\left\|R_{k+1}\right\|^{2}}{\left\|R_{k}\right\|^{2}} P_{k}, \\
Q_{k+1}=C_{1}^{H} R_{k+1} D_{1}^{H}+\bar{C}_{2}^{H} \overline{R_{k+1}} \bar{D}_{2}^{H} \\
+D_{3} R_{k+1}^{H} C_{3}+\overline{D_{4}} R_{k+1}^{T} \overline{C_{4}}+\frac{\left\|R_{k+1}\right\|^{2}}{\left\|R_{k}\right\|^{2}} Q_{k} ;
\end{aligned}
$$

(6) If $R_{k+1}=0$, then stop; else let $k=k+1$ go to Step 5 .

To prove the convergence property of Algorithm 3, we first establish the following basic properties.

Lemma 4. Suppose that the matrix equation (1) is consistent and $V^{*}, W^{*}$ are arbitrary solutions of (1). Then for any initial matrices $V_{1}$ and $W_{1}$, we have

$$
\operatorname{Re}\left\{\operatorname{tr}\left[P_{i}^{H}\left(V^{*}-V_{i}\right)+Q_{i}^{H}\left(W^{*}-W_{i}\right)\right]\right\}=\left\|R_{i}\right\|^{2},
$$

where the sequence $\left\{V_{i}\right\},\left\{P_{i}\right\},\left\{W_{i}\right\},\left\{Q_{i}\right\}$, and $\left\{R_{i}\right\}$ are generated by Algorithm 3 for $i=1,2, \ldots$.

Proof. We apply mathematical induction to prove the conclusion.
For $i=1$, from Algorithm 3 we have

$$
\begin{aligned}
& \operatorname{Re}\left\{\operatorname{tr}\left[P_{1}^{H}\left(V^{*}-V_{1}\right)+Q_{1}^{H}\left(W^{*}-W_{1}\right)\right]\right\} \\
& =\operatorname{Re}\left\{\operatorname { t r } \left[\left(A_{1}^{H} R_{1} B_{1}^{H}+\bar{A}_{2}^{H} \overline{R_{1}} \bar{B}_{2}^{H}\right.\right.\right. \\
& \left.+B_{3} R_{1}^{H} A_{3}+\overline{B_{4}} R_{1}^{T} \overline{A_{4}} \bar{B}_{2}^{H}\right)^{H}\left(V^{*}-V_{1}\right) \\
& +\left(C_{1}^{H} R_{1} D_{1}^{H}+\bar{C}_{2}^{H} \overline{R_{1}} \bar{D}_{2}^{H}\right. \\
& \left.+D_{3} R_{1}^{H} C_{3}+\overline{D_{4}} R_{1}^{T} \overline{C_{4}} \bar{D}_{2}^{H}\right)^{H} \\
& \left.\left.\quad \times\left(W^{*}-W_{1}\right)\right]\right\} \\
& =\operatorname{Re}\left\{\operatorname { t r } \left[R_{1}^{H} A_{1}\left(V^{*}-V_{1}\right) B_{1}+\bar{R}_{1}^{H} \overline{A_{2}}\left(V^{*}-V_{1}\right) \overline{B_{2}}\right.\right. \\
& +R_{1} B_{3}^{H}\left(V^{*}-V_{1}\right) A_{3}^{H}+\overline{R_{1}} \bar{B}_{4}^{H}\left(V^{*}-V_{1}\right) \bar{A}_{4}^{H} \\
& +R_{1}^{H} C_{1}\left(W^{*}-W_{1}\right) D_{1}+\bar{R}_{1}^{H} \overline{C_{2}}\left(W^{*}-W_{1}\right) \overline{D_{2}} \\
& +R_{1} D_{3}^{H}\left(W^{*}-W_{1}\right) C_{3}^{H} \\
& \left.\left.+\bar{R}_{1} \bar{D}_{4}^{H}\left(W^{*}-W_{1}\right) \bar{C}_{4}^{H}\right]\right\} .
\end{aligned}
$$

From properties of trace and conjugate

$$
\begin{aligned}
& \operatorname{Re}\left\{\operatorname{tr}\left[P_{1}^{H}\left(V^{*}-V_{1}\right)+Q_{1}^{H}\left(W^{*}-W_{1}\right)\right]\right\} \\
&=\operatorname{Re}\left\{\operatorname { t r } \left[R_{1}^{H} A_{1}\left(V^{*}-V_{1}\right) B_{1}+\overline{\bar{R}_{1}^{H} \overline{A_{2}}\left(V^{*}-V_{1}\right) \overline{B_{2}}}\right.\right. \\
&+A_{3}\left(V^{*}-V_{1}\right)^{H} B_{3} R_{1}^{H}+A_{4}\left(V^{*}-V_{1}\right)^{T} B_{4} R_{1}^{H} \\
&+R_{1}^{H} C_{1}\left(W^{*}-W_{1}\right) D_{1} \\
&+\overline{\overline{R_{1}}} \overline{C_{2}}\left(W^{*}-W_{1}\right) \overline{D_{2}} \\
&+ C_{3}\left(W^{*}-W_{1}\right)^{H} D_{3} R_{1}^{H} \\
&\left.\left.+C_{4}\left(W^{*}-W_{1}\right)^{T} D_{4} R_{1}^{H}\right]\right\} \\
&=\operatorname{Re}\left\{\operatorname { t r } \left[R_{1}^{H}\right.\right. A_{1}\left(V^{*}-V_{1}\right) B_{1}+R_{1}^{H} A_{2} \overline{\left(V^{*}-V_{1}\right)} B_{2} \\
&+R_{1}^{H} A_{3}\left(V^{*}-V_{1}\right)^{H} B_{3} \\
&+R_{1}^{H} A_{4}\left(V^{*}-V_{1}\right)^{T} B_{4} \\
&+R_{1}^{H} C_{1}\left(W^{*}-W_{1}\right) D_{1} \\
&+R_{1}^{H} C_{2} \overline{\left(W^{*}-W_{1}\right)} D_{2} \\
&+R_{1}^{H} C_{3}\left(W^{*}-W_{1}\right)^{H} D_{3} \\
&\left.\left.+R_{1}^{H} C_{4}\left(W^{*}-W_{1}\right)^{T} D_{4}\right]\right\}
\end{aligned}
$$




$$
\begin{aligned}
=\operatorname{Re}\left\{\operatorname { t r } \left[R _ { 1 } ^ { H } \left(A_{1}\right.\right.\right. & V^{*} B_{1}+C_{1} W^{*} D_{1}+A_{2} \overline{V^{*}} B_{2} \\
& +C_{2} \overline{W^{*}} D_{2}+A_{3} V^{* H} B_{3}+C_{3} W^{* H} D_{3} \\
& +A_{4} V^{* T} B_{4}+C_{4} W^{* T} D_{4}-A_{1} V_{1} B_{1} \\
& -C_{1} W_{1} D_{1}-A_{2} \overline{V_{1}} B_{2}-C_{2} \overline{W_{1}} D_{2} \\
& -A_{3} V_{1}^{H} B_{3}-C_{3} W_{1}^{H} D_{3} \\
& \left.\left.\left.-A_{4} V_{1}^{T} B_{4}-C_{4} W_{1}^{T} D_{4}\right)\right]\right\} .
\end{aligned}
$$

In view that $V^{*}$ and $W^{*}$ are solutions of matrix equation (1), with this relation we have

$$
\begin{gathered}
\operatorname{Re}\left\{\operatorname{tr}\left[P_{1}^{H}\left(V^{*}-V_{1}\right)+Q_{1}^{H}\left(W^{*}-W_{1}\right)\right]\right\} \\
=\operatorname{Re}\left\{\operatorname { t r } \left[R _ { 1 } ^ { H } \left(E-A_{1} V_{1} B_{1}-C_{1} W_{1} D_{1}\right.\right.\right. \\
-A_{2} \overline{V_{1}} B_{2}-C_{2} \overline{W_{1}} D_{2} \\
-A_{3} V_{1}^{H} B_{3}-C_{3} W_{1}^{H} D_{3} \\
\left.\left.\left.-A_{4} V_{1}^{T} B_{4}-C_{4} W_{1}^{T} D_{4}\right)\right]\right\} \\
=\operatorname{Re}\left\{\operatorname{tr}\left[R_{1}^{H} R_{1}\right]\right\}=\left\|R_{1}\right\|^{2} .
\end{gathered}
$$

This implies that (8) holds for $i=1$.

Assume that (8) holds for $=k$. That is,

$$
\operatorname{Re}\left\{\operatorname{tr}\left[P_{k}^{H}\left(V^{*}-V_{k}\right)+Q_{k}^{H}\left(W^{*}-W_{k}\right)\right]\right\}=\left\|R_{k}\right\|^{2} .
$$

Then we have to prove that the conclusion holds for $i=k+1$; it follows from Algorithm 3 that

$$
\begin{gathered}
\operatorname{Re}\left\{\operatorname{tr}\left[P_{k+1}^{H}\left(V^{*}-V_{k+1}\right)+Q_{k+1}^{H}\left(W^{*}-W_{k+1}\right)\right]\right\} \\
=\operatorname{Re}\left\{\operatorname { t r } \left[A_{1}^{H} R_{k+1} B_{1}^{H}+\bar{A}_{2}^{H} \overline{R_{k+1}} \bar{B}_{2}^{H}\right.\right. \\
+B_{3} R_{k+1}^{H} A_{3}+\overline{B_{4}} R_{k+1}^{T} \overline{A_{4}} \\
\left.+\frac{\left\|R_{k+1}\right\|^{2}}{\left\|R_{k}\right\|^{2}} P_{k}\right)^{H}\left(V^{*}-V_{k+1}\right) \\
\left.+\left(\begin{array}{c}
C_{1}^{H} R_{k+1} D_{1}^{H}+\bar{C}_{2}^{H} \overline{R_{k+1}} \bar{D}_{2}^{H} \\
+D_{3} R_{k+1}^{H} C_{3}+\overline{D_{4}} R_{k+1}^{T} \overline{C_{4}} \\
\left.+\frac{\left\|R_{k+1}\right\|^{2}}{\left\|R_{k}\right\|^{2}} Q_{k}\right)^{H}\left(W^{*}-W_{k+1}\right)
\end{array}\right]\right\}
\end{gathered}
$$

$$
\begin{aligned}
=\operatorname{Re}\left\{\operatorname { t r } \left[R_{k+1}^{H} A_{1}\left(V^{*}-V_{k+1}\right) B_{1}\right.\right. \\
+R_{k+1}^{T} \bar{A}_{2}\left(V^{*}-V_{k+1}\right) \bar{B}_{2} \\
+R_{k+1} B_{3}^{H}\left(V^{*}-V_{k+1}\right) A_{3}^{H} \\
+\overline{R_{k+1}}{\overline{B_{4}}}^{H}\left(V^{*}-V_{k+1}\right){\overline{A_{4}}}^{H} \\
+R_{k+1}^{H} C_{1}\left(W^{*}-W_{k+1}\right) D_{1} \\
+R_{k+1}^{T} \bar{C}_{2}\left(W^{*}-W_{k+1}\right) \bar{D}_{2} \\
+R_{k+1} D_{3}^{H}\left(W^{*}-W_{k+1}\right) C_{3}^{H} \\
+\bar{R}_{k+1} \bar{D}_{4}^{H}\left(W^{*}-W_{k+1}\right) \bar{C}_{4}^{H} \\
+\frac{\left\|R_{k+1}\right\|^{2}}{\left\|R_{k}\right\|^{2}}\left(P_{k}^{H}\left(V^{*}-V_{k+1}\right)\right.
\end{aligned}
$$

From properties of trace and conjugate we get

$$
\begin{aligned}
& \operatorname{Re}\left\{\operatorname{tr}\left[P_{k+1}^{H}\left(V^{*}-V_{k+1}\right)+Q_{k+1}^{H}\left(W^{*}-W_{k+1}\right)\right]\right\} \\
& =\operatorname{Re}\left\{\operatorname { t r } \left[R_{k+1}^{H} A_{1}\left(V^{*}-V_{k+1}\right) B_{1}\right.\right.
\end{aligned}
$$

$$
\begin{aligned}
& +\overline{R_{k+1}^{T} \bar{A}_{2}\left(V^{*}-V_{k+1}\right) \bar{B}_{2}} \\
& +A_{3}\left(V^{*}-V_{k+1}\right)^{H} B_{3} R_{k+1}^{H} \\
& +A_{4}\left(V^{*}-V_{k+1}\right)^{T} B_{4} R_{k+1}^{H} \\
& +R_{k+1}^{H} C_{1}\left(W^{*}-W_{k+1}\right) D_{1} \\
& +\overline{R_{k+1}^{T} \bar{C}_{2}\left(W^{*}-W_{k+1}\right) \bar{D}_{2}} \\
& +C_{3}\left(W^{*}-W_{k+1}\right)^{H} D_{3} R_{k+1}^{H} \\
& +C_{4}\left(W^{*}-W_{k+1}\right)^{T} D_{4} R_{k+1}^{H}+\frac{\left\|R_{k+1}\right\|^{2}}{\left\|R_{k}\right\|^{2}} \\
& +\left(P_{k}^{H}\left(V^{*}-V_{k+1}\right)\right. \\
& \left.\left.\left.+Q_{k}^{H}\left(W^{*}-W_{k+1}\right)\right)\right]\right\}
\end{aligned}
$$




$$
\begin{aligned}
&=\operatorname{Re}\{\operatorname{tr}[ R_{k+1}^{H} A_{1}\left(V^{*}-V_{k+1}\right) B_{1} \\
&+R_{k+1}^{H} A_{2} \overline{\left(V^{*}-V_{k+1}\right)} B_{2} \\
&+R_{k+1}^{H} A_{3}\left(V^{*}-V_{k+1}\right)^{H} B_{3} \\
&+R_{k+1}^{H} A_{4}\left(V^{*}-V_{k+1}\right)^{T} B_{4} \\
&+R_{k+1}^{H} C_{1}\left(W^{*}-W_{k+1}\right) D_{1} \\
&+R_{k+1}^{H} C_{2} \overline{\left(W^{*}-W_{k+1}\right)} D_{2} \\
&+R_{k+1}^{H} C_{3}\left(W^{*}-W_{k+1}\right)^{H} D_{3} \\
&\left.\left.+R_{k+1}^{H} C_{4}\left(W^{*}-W_{k+1}\right)^{T} D_{4}\right]\right\}+\frac{\left\|R_{k+1}\right\|^{2}}{\left\|R_{k}\right\|^{2}} \\
& \times \operatorname{Re}\left\{\operatorname { t r } \left[P_{k}^{H}\left(V^{*}-V_{k}-\frac{\left\|R_{k}\right\|^{2}}{\left\|P_{k}\right\|^{2}+\left\|Q_{k}\right\|^{2}} P_{k}\right)\right.\right. \\
& \quad+Q_{k}^{H}\left(W^{*}-W_{k}\right. \\
&\left.\left.\left.\quad \frac{\left\|R_{k}\right\|^{2}}{\left\|P_{k}\right\|^{2}+\left\|Q_{k}\right\|^{2}} Q_{k}\right)\right]\right\}
\end{aligned}
$$$$
=\operatorname{Re}\left\{\operatorname { t r } \left[R _ { k + 1 } ^ { H } \left(A_{1} V^{*} B_{1}+C_{1} W^{*} D_{1}\right.\right.\right.
$$$$
+A_{2} \overline{V^{*}} B_{2}+C_{2} \overline{W^{*}} D_{2}
$$$$
+A_{3} V^{* H} B_{3}+C_{3} W^{* H} D_{3}
$$$$
+A_{4} V^{* T} B_{4}+C_{4} W^{* T} D_{4}
$$$$
-A_{1} V_{k+1} B_{1}-C_{1} W_{k+1} D_{1}-A_{2} \overline{V_{k+1}} B_{2}
$$$$
-C_{2} \overline{W_{k+1}} D_{2}-A_{3} V_{k+1}^{H} B_{3}
$$$$
-C_{3} W_{k+1}^{H} D_{3}-A_{4} V_{k+1}^{T} B_{4}
$$$$
\left.\left.\left.-C_{4} W_{k+1}^{T} D_{4}\right)\right]\right\}
$$$$
+\frac{\left\|R_{k+1}\right\|^{2}}{\left\|R_{k}\right\|^{2}} \operatorname{Re}\left\{\operatorname { t r } \left[P_{k}^{H}\left(V^{*}-V_{k}\right)+Q_{k}^{H}\left(W^{*}-W_{k}\right)\right.\right.
$$$$
\left.\left.-\frac{\left\|R_{k}\right\|^{2}}{\left\|P_{k}\right\|^{2}+\left\|Q_{k}\right\|^{2}}\left(P_{k}^{H} P_{k}+Q_{k}^{H} Q_{k}\right)\right]\right\} .
$$

$$
\begin{gathered}
-A_{3} V_{k+1}^{H} B_{3}-C_{3} W_{k+1}^{H} D_{3} \\
\left.\left.\left.-A_{4} V_{k+1}^{T} B_{4}-C_{4} W_{k+1}^{T} D_{4}\right)\right]\right\} \\
+\frac{\left\|R_{k+1}\right\|^{2}}{\left\|R_{k}\right\|^{2}}\left[\left\|R_{k}\right\|^{2}-\frac{\left\|R_{k}\right\|^{2}}{\left\|P_{k}\right\|^{2}+\left\|Q_{k}\right\|^{2}}\left(\left\|P_{k}\right\|^{2}+\left\|Q_{k}\right\|^{2}\right)\right] \\
=\operatorname{Re}\left\{\operatorname{tr}\left[R_{k+1}^{H} R_{k+1}\right]\right\}=\left\|R_{k+1}\right\|^{2} .
\end{gathered}
$$

Then relation (8) holds by mathematical induction.

Lemma 5. Suppose that the matrix equation (1) is consistent and the sequences $\left\{R_{i}\right\},\left\{P_{i}\right\}$, and $\left\{Q_{i}\right\}$ are generated by Algorithm 3 with any initial matrices $V_{1}, W_{1}$, such that $R_{i} \neq 0$ for all $i=1,2, \ldots, k$, and then

$$
\begin{gathered}
\operatorname{Re}\left\{\operatorname{tr}\left(R_{j}^{H} R_{i}\right)\right\}=0, \\
\operatorname{Re}\left\{\operatorname{tr}\left(P_{j}^{H} P_{i}+Q_{j}^{H} Q_{i}\right)\right\}=0, \quad i, j=1,2, \ldots, k, i \neq j .
\end{gathered}
$$

Proof. We apply mathematical induction.

Step 1 . We prove that

$$
\begin{gathered}
\operatorname{Re}\left\{\operatorname{tr}\left(R_{i+1}^{H} R_{i}\right)\right\}=0, \\
\operatorname{Re}\left\{\operatorname{tr}\left(P_{i+1}^{H} P_{i}+Q_{i+1}^{H} Q_{i}\right)\right\}=0, \quad i=1,2, \ldots, k .
\end{gathered}
$$

First from Algorithm 3 we have

$$
\begin{aligned}
R_{k+1}= & E-A_{1} V_{k+1} B_{1}-C_{1} W_{k+1} D_{1}-A_{2} \overline{V_{k+1}} B_{2} \\
& -C_{2} \bar{W}_{k+1} D_{2}-A_{3} V_{k+1}^{H} B_{3} \\
& -C_{3} W_{k+1}^{H} D_{3}-A_{4} V_{k+1}^{T} B_{4}-C_{4} W_{k+1}^{T} D_{4} \\
R_{k+1}= & E-A_{1}\left(V_{k}+\frac{\left\|R_{k}\right\|^{2}}{\left\|P_{k}\right\|^{2}+\left\|Q_{k}\right\|^{2}} P_{k}\right) B_{1} \\
& -C_{1}\left(W_{k}+\frac{\left\|R_{k}\right\|^{2}}{\left\|P_{k}\right\|^{2}+\left\|Q_{k}\right\|^{2}} Q_{k}\right) D_{1} \\
& -A_{2}\left(V_{k}+\frac{\left\|R_{k}\right\|^{2}}{\left\|P_{k}\right\|^{2}+\left\|Q_{k}\right\|^{2}} P_{k}\right) B_{2} \\
& -C_{2}\left(W_{k}+\frac{\left\|R_{k}\right\|^{2}}{\left\|P_{k}\right\|^{2}+\left\|Q_{k}\right\|^{2}} Q_{k}\right) D_{2} \\
& -A_{3}\left(V_{k}+\frac{\left\|R_{k}\right\|^{2}}{\left\|P_{k}\right\|^{2}+\left\|Q_{k}\right\|^{2}} P_{k}\right)^{H} B_{3} \\
& -C_{3}\left(W_{k}+\frac{\left\|R_{k}\right\|^{2}}{\left\|P_{k}\right\|^{2}+\left\|Q_{k}\right\|^{2}} Q_{k}\right)^{H} D_{3} \\
& -A_{4}\left(V_{k}+\frac{\left\|R_{k}\right\|^{2}}{\left\|P_{k}\right\|^{2}+\left\|Q_{k}\right\|^{2}} P_{k}\right)^{T} B_{4} \\
B^{2} &
\end{aligned}
$$

In view that $V^{*}$ and $W^{*}$ are solutions of matrix equation (1), with relation (14) one has

$$
\begin{array}{r}
\operatorname{Re}\left\{\operatorname{tr}\left[P_{k+1}^{H}\left(V^{*}-V_{k+1}\right)+Q_{k+1}^{H}\left(W^{*}-W_{k+1}\right)\right]\right\} \\
=\operatorname{Re}\left\{\operatorname { t r } \left[R _ { k + 1 } ^ { H } \left(E-A_{1} V_{k+1} B_{1}-C_{1} W_{k+1} D_{1}\right.\right.\right. \\
-A_{2} \overline{V_{k+1}} B_{2}-C_{2} \overline{W_{k+1}} D_{2}
\end{array}
$$




$$
\begin{gathered}
-C_{4}\left(W_{k}+\frac{\left\|R_{k}\right\|^{2}}{\left\|P_{k}\right\|^{2}+\left\|Q_{k}\right\|^{2}} Q_{k}\right)^{T} D_{4} \\
=E-A_{1} V_{k} B_{1}-C_{1} W_{k} D_{1}-A_{2} \overline{V_{k}} B_{2}-C_{2} \overline{W_{k}} D_{2} \\
-A_{3} V_{k}^{H} B_{3}-C_{3} W_{k}^{H} D_{3}-A_{4} V_{k}^{T} B_{4} \\
-C_{4} W_{k}^{T} D_{4}-\frac{\left\|R_{k}\right\|^{2}}{\left\|P_{k}\right\|^{2}+\left\|Q_{k}\right\|^{2}} \\
\times\left(A_{1} P_{k} B_{1}+C_{1} Q_{k} D_{1}+A_{2} \overline{P_{k}} B_{2}\right. \\
+C_{2} \overline{Q_{k}} D_{2}+A_{3} P_{k}^{H} B_{3} \\
\left.+C_{3} Q_{k}^{H} D_{3}+A_{4} P_{k}^{T} B_{4}+C_{4} Q_{k}^{T} D_{4}\right), \\
R_{k+1}=R_{k}-\frac{\left\|R_{k}\right\|^{2}}{\left\|P_{k}\right\|^{2}+\left\|Q_{k}\right\|^{2}} \\
\times\left(A_{1} P_{k} B_{1}+C_{1} Q_{k} D_{1}+A_{2} \overline{P_{k}} B_{2}\right. \\
+C_{2} \overline{Q_{k}} D_{2}+A_{3} P_{k}^{H} B_{3} \\
\left.+C_{3} Q_{k}^{H} D_{3}+A_{4} P_{k}^{T} B_{4}+C_{4} Q_{k}^{T} D_{4}\right) .
\end{gathered}
$$

For $i=1$, it follows from (19) that

$$
\begin{aligned}
& \operatorname{Re}\left\{\operatorname{tr}\left(R_{2}^{H} R_{1}\right)\right\} \\
& =\operatorname{Re}\left\{\operatorname { t r } \left[\left(R_{1}-\frac{\left\|R_{1}\right\|^{2}}{\left\|P_{1}\right\|^{2}+\left\|Q_{1}\right\|^{2}}\right.\right.\right. \\
& \times\left(A_{1} P_{1} B_{1}+C_{1} Q_{1} D_{1}\right. \\
& +A_{2} \overline{P_{1}} B_{2}+C_{2} \overline{Q_{1}} D_{2} \\
& +A_{3} P_{1}^{H} B_{3}+C_{3} Q_{1}^{H} D_{3} \\
& \left.\left.\left.\left.+A_{4} P_{1}^{T} B_{4}+C_{4} Q_{1}^{T} D_{4}\right)\right)^{H} R_{1}\right]\right\} \\
& =\operatorname{Re}\left\{\operatorname{tr}\left(R_{1}^{H} R_{1}\right)-\frac{\left\|R_{1}\right\|^{2}}{\left\|P_{1}\right\|^{2}+\left\|Q_{1}\right\|^{2}}\right. \\
& \times \operatorname{tr}\left(P_{1}^{H} A_{1}^{H} R_{1} B_{1}^{H}+Q_{1}^{H} C_{1}^{H} R_{1} D_{1}^{H}\right. \\
& +\bar{P}_{1}{ }^{H} A_{2}^{H} R_{1} B_{2}^{H}+{\overline{Q_{1}}}^{H} C_{2}^{H} R_{1} D_{2}^{H} \\
& +P_{1} A_{3}^{H} R_{1} B_{3}^{H}+Q_{1} C_{3}^{H} R_{1} D_{3}^{H} \\
& \left.\left.+\overline{P_{1}} A_{4}^{H} R_{1} B_{4}^{H}+\overline{Q_{1}} C_{4}^{H} R_{1} D_{4}^{H}\right)\right\}
\end{aligned}
$$

$$
\begin{aligned}
& =\left\|R_{1}\right\|^{2}-\frac{\left\|R_{1}\right\|^{2}}{\left\|P_{1}\right\|^{2}+\left\|Q_{1}\right\|^{2}} \\
& \times \operatorname{Re}\left\{\operatorname { t r } \left[P_{1}^{H} A_{1}^{H} R_{1} B_{1}^{H}+Q_{1}^{H} C_{1}^{H} R_{1} D_{1}^{H}\right.\right. \\
& +\overline{{\overline{P_{1}}}^{H} A_{2}^{H} R_{1} B_{2}^{H}}+\overline{{\overline{Q_{1}}}^{H} C_{2}^{H} R_{1} D_{2}^{H}} \\
& +B_{3} R_{1}^{H} A_{3} P_{1}^{H}+D_{3} R_{1}^{H} C_{3} Q_{1}^{H} \\
& \left.\left.+\overline{B_{4}} R_{1}^{T} \overline{A_{4}} P_{1}^{H}+\overline{D_{4}} R_{1}^{T} \overline{C_{4}} Q_{1}^{H}\right]\right\} \\
& =\left\|R_{1}\right\|^{2}-\frac{\left\|R_{1}\right\|^{2}}{\left\|P_{1}\right\|^{2}+\left\|Q_{1}\right\|^{2}} \\
& \times \operatorname{Re}\left\{\operatorname { t r } \left[P _ { 1 } ^ { H } \left(A_{1}^{H} R_{1} B_{1}^{H}+\bar{A}_{2}^{H} \bar{R}_{1} \bar{B}_{2}^{H}\right.\right.\right. \\
& \left.+B_{3} R_{1}^{H} A_{3}+\overline{B_{4}} R_{1}^{T} \overline{A_{4}}\right) \\
& +Q_{1}^{H}\left(C_{1}^{H} R_{1} D_{1}^{H}+\bar{C}_{2}^{H} \bar{R}_{1} \bar{D}_{2}^{H}\right. \\
& \left.\left.\left.+D_{3} R_{1}^{H} C_{3}+\overline{D_{4}} R_{1}^{T} \overline{C_{4}}\right)\right]\right\}
\end{aligned}
$$$$
=\left\|R_{1}\right\|^{2}-\frac{\left\|R_{1}\right\|^{2}}{\left\|P_{1}\right\|^{2}+\left\|Q_{1}\right\|^{2}} \operatorname{Re}\left\{\operatorname{tr}\left(P_{1}^{H} P_{1}+Q_{1}^{H} Q_{1}\right)\right\},
$$$$
\operatorname{Re}\left\{\operatorname{tr}\left(R_{2}^{H} R_{1}\right)\right\}
$$$$
=\left\|R_{1}\right\|^{2}-\frac{\left\|R_{1}\right\|^{2}}{\left\|P_{1}\right\|^{2}+\left\|Q_{1}\right\|^{2}}\left(\left\|P_{1}\right\|^{2}+\left\|Q_{1}\right\|^{2}\right)=0 .
$$

This implies that (17) is satisfied for $i=1$.

From Algorithm 3 we have

$$
\begin{array}{r}
\operatorname{Re}\left\{\operatorname{tr}\left(P_{2}^{H} P_{1}+Q_{2}^{H} Q_{1}\right)\right\} \\
=\operatorname{Re}\left\{\operatorname { t r } \left[\left(A_{1}^{H} R_{2} B_{1}^{H}+\bar{A}_{2}^{H} \overline{R_{2}} \bar{B}_{2}^{H}+B_{3} R_{2}^{H} A_{3}\right.\right.\right. \\
\left.+\overline{B_{4}} R_{2}^{T} \overline{A_{4}}+\frac{\left\|R_{2}\right\|^{2}}{\left\|R_{1}\right\|^{2}} P_{1}\right)^{H} P_{1} \\
+\left(C_{1}^{H} R_{2} D_{1}^{H}+\bar{C}_{2}^{H} \overline{R_{2}} \bar{D}_{2}^{H}+D_{3} R_{2}^{H} C_{3}\right. \\
\left.\left.\left.+\overline{D_{4}} R_{2}^{T} \overline{C_{4}}+\frac{\left\|R_{2}\right\|^{2}}{\left\|R_{1}\right\|^{2}} Q_{1}\right)^{H} Q_{1}\right]\right\}
\end{array}
$$




$$
\begin{aligned}
& =\operatorname{Re}\left\{\operatorname { t r } \left[R_{2}^{H} A_{1} P_{1} B_{1}+\bar{R}_{2}{ }^{H} \bar{A}_{2} P_{1} \bar{B}_{2}+R_{2} B_{3}^{H} P_{1} A_{3}^{H}\right.\right. \\
& +\overline{R_{2}}{\overline{B_{4}}}^{H} P_{1}{\overline{A_{4}}}^{H}+\frac{\left\|R_{2}\right\|^{2}}{\left\|R_{1}\right\|^{2}} P_{1}^{H} P_{1}+R_{2}^{H} C_{1} Q_{1} D_{1} \\
& +{\overline{R_{2}}}^{H} \bar{C}_{2} Q_{1} \bar{D}_{2}+R_{2} D_{3}^{H} Q_{1} C_{3}^{H} \\
& \left.\left.+\overline{R_{2}}{\overline{D_{4}}}^{H} Q_{1}{\overline{C_{4}}}^{H}+\frac{\left\|R_{2}\right\|^{2}}{\left\|R_{1}\right\|^{2}} Q_{1}^{H} Q_{1}\right]\right\} \\
& =\operatorname{Re}\left\{\operatorname { t r } \left[R_{2}^{H} A_{1} P_{1} B_{1}+\overline{\bar{R}_{2}{ }^{H} \bar{A}_{2} P_{1} \bar{B}_{2}}+A_{3} P_{1}^{H} B_{3} R_{2}^{H}\right.\right. \\
& +A_{4} P_{1}^{T} B_{4} R_{2}^{H}+R_{2}^{H} C_{1} Q_{1} D_{1} \\
& +\overline{\bar{R}_{2}{ }^{H} \bar{C}_{2} Q_{1} \bar{D}_{2}}+C_{3} Q_{1}^{H} D_{3} R_{2}^{H} \\
& \left.\left.+C_{4} Q_{1}^{T} D_{4} R_{2}^{H}+\frac{\left\|R_{2}\right\|^{2}}{\left\|R_{1}\right\|^{2}}\left(P_{1}^{H} P_{1}+Q_{1}^{H} Q_{1}\right)\right]\right\} \\
& =\operatorname{Re}\left\{\operatorname { t r } \left[R_{2}^{H} A_{1} P_{1} B_{1}+R_{2}^{H} A_{2} \bar{P}_{1} B_{2}+R_{2}^{H} A_{3} P_{1}^{H} B_{3}\right.\right. \\
& +R_{2}^{H} A_{4} P_{1}^{T} B_{4}+R_{2}^{H} C_{1} Q_{1} D_{1} \\
& +R_{2}^{H} C_{2} \overline{Q_{1}} D_{2}+R_{2}^{H} C_{3} Q_{1}^{H} D_{3} \\
& \left.\left.+R_{2}^{H} C_{4} Q_{1}^{T} D_{4}+\frac{\left\|R_{2}\right\|^{2}}{\left\|R_{1}\right\|^{2}}\left(P_{1}^{H} P_{1}+Q_{1}^{H} Q_{1}\right)\right]\right\} \\
& =\operatorname{Re}\left\{\operatorname { t r } \left[R _ { 2 } ^ { H } \left(A_{1} P_{1} B_{1}+C_{1} Q_{1} D_{1}+A_{2} \overline{P_{1}} B_{2}\right.\right.\right. \\
& +C_{2} \overline{Q_{1}} D_{2}+A_{3} P_{1}^{H} B_{3}+C_{3} Q_{1}^{H} D_{3} \\
& \left.\left.\left.+A_{4} P_{1}^{T} B_{4}+C_{4} Q_{1}^{H} D_{4}\right)\right]\right\} \\
& +\frac{\left\|R_{2}\right\|^{2}}{\left\|R_{1}\right\|^{2}}\left(\left\|P_{1}\right\|^{2}+\left\|Q_{1}\right\|^{2}\right) \\
& =\frac{\left\|P_{1}\right\|^{2}+\left\|Q_{1}\right\|^{2}}{\left\|R_{1}\right\|^{2}} \operatorname{Re}\left\{\operatorname{tr}\left[R_{2}^{H}\left(R_{1}-R_{2}\right)\right]\right\} \\
& +\frac{\left\|R_{2}\right\|^{2}}{\left\|R_{1}\right\|^{2}}\left(\left\|P_{1}\right\|^{2}+\left\|Q_{1}\right\|^{2}\right) \\
& =-\frac{\left\|P_{1}\right\|^{2}+\left\|Q_{1}\right\|^{2}}{\left\|R_{1}\right\|^{2}}\left(\left\|R_{2}\right\|^{2}\right) \\
& +\frac{\left\|R_{2}\right\|^{2}}{\left\|R_{1}\right\|^{2}}\left(\left\|P_{1}\right\|^{2}+\left\|Q_{1}\right\|^{2}\right)=0 .
\end{aligned}
$$

This implies that (18) is satisfied for $i=1$.
Assume that (17) and (18) hold for $i=k-1$, from Algorithm 3 we have

$$
\begin{aligned}
& \operatorname{Re}\left\{\operatorname{tr}\left(R_{k+1}^{H} R_{k}\right)\right\} \\
& =\operatorname{Re}\left\{\operatorname { t r } \left[\left(R_{k}-\frac{\left\|R_{k}\right\|^{2}}{\left\|P_{k}\right\|^{2}+\left\|Q_{k}\right\|^{2}}\right.\right.\right. \\
& \times\left(A_{1} P_{k} B_{1}+C_{1} Q_{k} D_{1}+A_{2} \overline{P_{k}} B_{2}\right. \\
& +C_{2} \overline{Q_{k}} D_{2}+A_{3} P_{k}^{H} B_{3}+C_{3} Q_{k}^{H} D_{3} \\
& \left.\left.\left.\left.+A_{4} P_{k}^{T} B_{4}+C_{4} Q_{k}^{T} D_{4}\right)\right)^{H} R_{k}\right]\right\},
\end{aligned}
$$

$\operatorname{Re}\left\{\operatorname{tr}\left(R_{k+1}^{H} R_{k}\right)\right\}$

$$
\begin{aligned}
=\operatorname{Re}\left\{\operatorname{tr}\left(R_{k}^{H} R_{k}\right)\right. & -\frac{\left\|R_{k}\right\|^{2}}{\left\|P_{k}\right\|^{2}+\left\|Q_{k}\right\|^{2}} \\
\times \operatorname{tr}( & P_{k}^{H} A_{1}^{H} R_{k} B_{1}^{H}+Q_{k}^{H} C_{1}^{H} R_{k} D_{1}^{H} \\
& +{\overline{P_{k}}}^{H} A_{2}^{H} R_{k} B_{2}^{H}+\overline{Q_{k}}{ }^{H} C_{2}^{H} R_{k} D_{2}^{H} \\
& +P_{k} A_{3}^{H} R_{k} B_{3}^{H}+Q_{k} C_{3}^{H} R_{k} D_{3}^{H} \\
& \left.\left.+\overline{P_{k}} A_{4}^{H} R_{k} B_{4}^{H}+\overline{Q_{k}} C_{4}^{H} R_{k} D_{4}^{H}\right)\right\}
\end{aligned}
$$$$
=\left\|R_{k}\right\|^{2}-\frac{\left\|R_{k}\right\|^{2}}{\left\|P_{k}\right\|^{2}+\left\|Q_{k}\right\|^{2}}
$$$$
\times \operatorname{Re}\left\{\operatorname { t r } \left(P_{k}^{H} A_{1}^{H} R_{k} B_{1}^{H}+Q_{k}^{H} C_{1}^{H} R_{k} D_{1}^{H}\right.\right.
$$$$
+\overline{{\overline{P_{k}}}^{H} A_{2}^{H} R_{k} B_{2}^{H}}+\overline{{\overline{Q_{k}}}^{H} C_{2}^{H} R_{k} D_{2}^{H}}
$$$$
+B_{3} R_{k}^{H} A_{3} P_{k}^{H}+D_{3} R_{k}^{H} C_{3} Q_{k}^{H}
$$$$
\left.\left.+\overline{B_{4}} R_{k}^{T} \overline{A_{4}} P_{k}^{H}+\overline{D_{4}} R_{k}^{T} \overline{C_{4}} Q_{k}^{H}\right)\right\}
$$$$
=\left\|R_{1}\right\|^{2}-\frac{\left\|R_{1}\right\|^{2}}{\left\|P_{k}\right\|^{2}+\left\|Q_{k}\right\|^{2}}
$$$$
\times \operatorname{Re}\left\{\operatorname { t r } \left[P _ { k } ^ { H } \left(A_{1}^{H} R_{k} B_{1}^{H}+\bar{A}_{2}^{H} \overline{R_{k}} \bar{B}_{2}^{H}\right.\right.\right.
$$$$
\left.+B_{3} R_{k}^{H} A_{3}+\overline{B_{4}} R_{k}^{T} \overline{A_{4}}\right)
$$$$
+Q_{k}^{H}\left(C_{1}^{H} R_{k} D_{1}^{H}+\bar{C}_{2}^{H}{\overline{R_{k}}}_{D_{2}^{H}}^{H}\right.
$$$$
\left.\left.\left.+D_{3} R_{k}^{H} C_{3}+\overline{D_{4}} R_{k}^{T} \overline{C_{4}}\right)\right]\right\}
$$ 


$$
\begin{gathered}
=\left\|R_{k}\right\|^{2}-\frac{\left\|R_{k}\right\|^{2}}{\left\|P_{k}\right\|^{2}+\left\|Q_{k}\right\|^{2}} \\
\times \operatorname{Re}\left\{\operatorname { t r } \left[P_{k}^{H}\left(P_{k}-\frac{\left\|R_{k}\right\|^{2}}{\left\|R_{k-1}\right\|^{2}} P_{k-1}\right)\right.\right. \\
\left.\left.+Q_{k}^{H}\left(Q_{k}-\frac{\left\|R_{k}\right\|^{2}}{\left\|R_{k-1}\right\|^{2}} Q_{k-1}\right)\right]\right\} \\
=\left\|R_{k}\right\|^{2}-\frac{\left\|R_{k}\right\|^{2}}{\left\|P_{k}\right\|^{2}+\left\|Q_{k}\right\|^{2}}\left(\left\|P_{k}\right\|^{2}+\left\|Q_{k}\right\|^{2}\right)=0 .
\end{gathered}
$$

Thus (17) holds for $i=k$.

Also, from Algorithm 3 we have

$$
\begin{aligned}
& \operatorname{Re}\left\{\operatorname{tr}\left(P_{k+1}^{H} P_{k}+Q_{k+1}^{H} Q_{k}\right)\right\} \\
& =\operatorname{Re}\left\{\operatorname { t r } \left[\left(A_{1}^{H} R_{k+1} B_{1}^{H}+\bar{A}_{2}^{H} \overline{R_{k+1}} \bar{B}_{2}^{H}\right.\right.\right. \\
& +B_{3} R_{k+1}^{H} A_{3}+\overline{B_{4}} R_{k+1}^{T} \overline{A_{4}} \\
& \left.+\frac{\left\|R_{k+1}\right\|^{2}}{\left\|R_{k}\right\|^{2}} P_{k}\right)^{H} P_{k} \\
& +\left(C_{1}^{H} R_{k+1} D_{1}^{H}+\bar{C}_{2}^{H} \overline{R_{k+1}} \bar{D}_{2}^{H}\right. \\
& +D_{3} R_{k+1}^{H} C_{3}+\overline{D_{4}} R_{k+1}^{T} \overline{C_{4}} \\
& \left.\left.\left.+\frac{\left\|R_{k+1}\right\|^{2}}{\left\|R_{k}\right\|^{2}} Q_{k}\right)^{H} Q_{k}\right]\right\} \\
& =\operatorname{Re}\left\{\operatorname { t r } \left[R_{k+1}^{H} A_{1} P_{k} B_{1}+\overline{R_{k+1}} H \bar{A}_{2} P_{k} \bar{B}_{2}\right.\right. \\
& +R_{k+1} B_{3}^{H} P_{k} A_{3}^{H}+\overline{R_{k+1}}{\overline{B_{4}}}^{H} P_{k}{\overline{A_{4}}}^{H} \\
& +\frac{\left\|R_{k+1}\right\|^{2}}{\left\|R_{k}\right\|^{2}} P_{k}^{H} P_{k}+R_{k+1}^{H} C_{1} Q_{k} D_{1} \\
& +{\overline{R_{k+1}}}^{H} \bar{C}_{2} Q_{k} \bar{D}_{2}+R_{k+1} D_{3}^{H} Q_{k} C_{3}^{H} \\
& \left.\left.+\overline{R_{k+1}}{\overline{D_{4}}}^{H} Q_{k}{\overline{C_{4}}}^{H}+\frac{\left\|R_{k+1}\right\|^{2}}{\left\|R_{k}\right\|^{2}} Q_{k}^{H} Q_{k}\right]\right\}
\end{aligned}
$$

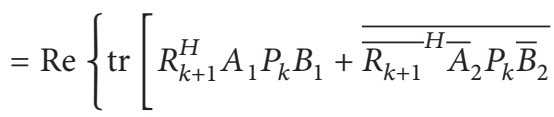

$$
\begin{aligned}
& +A_{3} P_{k}^{H} B_{3} R_{k+1}^{H}+A_{4} P_{k}^{T} B_{4} R_{k+1}^{H}
\end{aligned}
$$

$$
\begin{aligned}
& +\frac{\left\|R_{k+1}\right\|^{2}}{\left\|R_{k}\right\|^{2}} P_{k}^{H} P_{k}+R_{k+1}^{H} C_{1} Q_{k} D_{1}
\end{aligned}
$$

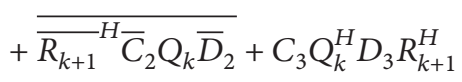

$$
\begin{aligned}
& \left.\left.+C_{4} Q_{k}^{T} D_{4} R_{k+1}^{H}+\frac{\left\|R_{k+1}\right\|^{2}}{\left\|R_{k}\right\|^{2}} Q_{k}^{H} Q_{k}\right]\right\} \\
& =\operatorname{Re}\left\{\operatorname { t r } \left[R _ { k + 1 } ^ { H } \left(A_{1} P_{k} B_{1}+C_{1} Q_{k} D_{1}\right.\right.\right. \\
& +A_{2} \overline{P_{k}} B_{2}+C_{2} \overline{Q_{k}} D_{2}+A_{3} P_{k}^{H} B_{3} \\
& \left.+C_{3} Q_{k}^{H} D_{3}+A_{4} P_{k}^{T} B_{4}+C_{4} Q_{k}^{T} D_{4}\right) \\
& \left.\left.+\frac{\left\|R_{k+1}\right\|^{2}}{\left\|R_{k}\right\|^{2}}\left(P_{k}^{H} P_{k}+Q_{k}^{H} Q_{k}\right)\right]\right\} \\
& =\frac{\left\|P_{k}\right\|^{2}+\left\|Q_{k}\right\|^{2}}{\left\|R_{k}\right\|^{2}} \operatorname{Re}\left\{\operatorname{tr}\left(R_{k+1}^{H}\left(R_{k}-R_{k+1}\right)\right)\right\} \\
& +\frac{\left\|R_{k+1}\right\|^{2}}{\left\|R_{k}\right\|^{2}}\left(\left\|P_{k}\right\|^{2}+\left\|Q_{k}\right\|^{2}\right) \\
& =-\frac{\left\|P_{k}\right\|^{2}+\left\|Q_{k}\right\|^{2}}{\left\|R_{k}\right\|^{2}}\left(\left\|R_{k+1}\right\|^{2}\right) \\
& +\frac{\left\|R_{k+1}\right\|^{2}}{\left\|R_{k}\right\|^{2}}\left(\left\|P_{k}\right\|^{2}+\left\|Q_{k}\right\|^{2}\right)=0 .
\end{aligned}
$$

This implies that (17) and (18) hold for $i=k$.

Then relations (17) and (18) holds by mathematical induction.

Step 2. We want to show that

$$
\begin{gathered}
\operatorname{Re}\left(\operatorname{tr}\left(R_{i+l}^{H} R_{i}\right)\right)=0, \\
\operatorname{Re}\left(\operatorname{tr}\left(P_{i+l}^{H} P_{i}+Q_{i+l}^{H} Q_{i}\right)\right)=0
\end{gathered}
$$

holds for $l \geq 1$. We will prove this conclusion by induction. The case of $l=1$ has been proven in Step 1 . Now we assume that (24) holds for $l \leq s, s \geq 1$. The aim is to show that

$$
\begin{gathered}
\operatorname{Re}\left(\operatorname{tr}\left(R_{i+s+1}^{H} R_{i}\right)\right)=0, \\
\operatorname{Re}\left(\operatorname{tr}\left(P_{i+s+1}^{H} P_{i}+Q_{i+s+1}^{H} Q_{i}\right)\right)=0 .
\end{gathered}
$$

First we prove the following:

$$
\begin{gathered}
\operatorname{Re}\left(\operatorname{tr}\left(R_{s+1}^{H} R_{0}\right)\right)=0, \\
\operatorname{Re}\left(\operatorname{tr}\left(P_{s+1}^{H} P_{0}+Q_{s+1}^{H} Q_{0}\right)\right)=0 .
\end{gathered}
$$


By using Algorithm 3, from (19) and induction we have

$$
\begin{gathered}
\operatorname{Re}\left\{\operatorname{tr}\left(R_{s+1}^{H} R_{0}\right)\right\} \\
=\operatorname{Re}\left\{\operatorname { t r } \left[\left(R_{s}-\frac{\left\|R_{s}\right\|^{2}}{\left\|P_{s}\right\|^{2}+\left\|Q_{s}\right\|^{2}}\right.\right.\right. \\
\times\left(A_{1} P_{s} B_{1}+C_{1} Q_{s} D_{1}+A_{2} \overline{P_{s}} B_{2}\right. \\
+C_{2} \overline{Q_{s}} D_{2}+A_{3} P_{s}^{H} B_{3}+C_{3} Q_{s}^{H} D_{3} \\
\left.\left.\left.\left.+A_{4} P_{s}^{T} B_{4}+C_{4} Q_{s}^{T} D_{4}\right)\right)^{H} R_{0}\right]\right\} \\
\operatorname{Re}\left\{\begin{array}{c}
\operatorname{tr}\left(R_{s}^{H} R_{0}\right)-\frac{\left\|R_{s}\right\|^{2}}{\left\|P_{s}\right\|^{2}+\left\|Q_{s}\right\|^{2}} \\
\times \operatorname{tr}\left(P_{s}^{H} A_{1}^{H} R_{0} B_{1}^{H}+Q_{s}^{H} C_{1}^{H} R_{0} D_{1}^{H}\right. \\
+\bar{P}_{s}^{H} A_{2}^{H} R_{0} B_{2}^{H}+{\overline{Q_{s}}}^{H} C_{2}^{H} R_{0} D_{2}^{H} \\
+P_{s} A_{3}^{H} R_{0} B_{3}^{H}+Q_{s} C_{3}^{H} R_{0} D_{3}^{H} \\
\left.\left.+\overline{P_{s}} A_{4}^{H} R_{0} B_{4}^{H}+\overline{Q_{s}} C_{4}^{H} R_{0} D_{4}^{H}\right)\right\},
\end{array}\right.
\end{gathered}
$$

$\operatorname{Re}\left\{\operatorname{tr}\left(R_{s+1}^{H} R_{0}\right)\right\}$

$$
\begin{gathered}
=\operatorname{Re}\left\{\operatorname{tr}\left(R_{s}^{H} R_{0}\right)\right\}-\frac{\left\|R_{s}\right\|^{2}}{\left\|P_{s}\right\|^{2}+\left\|Q_{s}\right\|^{2}} \\
\times \operatorname{Re}\left\{\operatorname { t r } \left(P_{s}^{H} A_{1}^{H} R_{0} B_{1}^{H}+Q_{s}^{H} C_{1}^{H} R_{0} D_{1}^{H}\right.\right. \\
+\overline{\bar{P}_{s}^{H} A_{2}^{H} R_{0} B_{2}^{H}}+\overline{\bar{Q}_{s}^{H} C_{2}^{H} R_{0} D_{2}^{H}} \\
+B_{3} R_{0}^{H} A_{3} P_{s}^{H}+D_{3} R_{0}^{H} C_{3} Q_{s}^{H} \\
\left.\left.+\overline{B_{4}} R_{0}^{T} \overline{A_{4}} P_{s}^{H}+\overline{D_{4}} R_{0}^{T} \overline{C_{4}} Q_{s}^{H}\right)\right\} \\
=-\frac{\left\|R_{s}\right\|^{2}}{\left\|P_{s}\right\|^{2}+\left\|Q_{s}\right\|^{2}} \\
\times \operatorname{Re}\left\{\operatorname { t r } \left(P _ { s } ^ { H } \left(A_{1}^{H} R_{0} B_{1}^{H}+\bar{A}_{2}^{H} \overline{R_{0}} \bar{B}_{2}^{H}\right.\right.\right. \\
\left.\quad+B_{3} R_{0}^{H} A_{3}+\overline{B_{4}} R_{0}^{T} \overline{A_{4}}\right) \\
+Q_{s}^{H}\left(C_{1}^{H} R_{0} D_{1}^{H}+\bar{C}_{2}^{H} \overline{R_{0}} \bar{D}_{2}^{H}\right. \\
\left.\left.\left.+D_{3} R_{0}^{H} C_{3}+\overline{D_{4}} R_{0}^{T} \overline{C_{4}}\right)\right)\right\} \\
=-\frac{\left\|R_{s}\right\|^{2}}{\left\|P_{s}\right\|^{2}+\left\|Q_{s}\right\|^{2}} \operatorname{Re}\left\{\operatorname{tr}\left(P_{s}^{H} P_{0}+Q_{s}^{H} Q_{0}\right)\right\}=0,
\end{gathered}
$$

$\operatorname{Re}\left\{\operatorname{tr}\left(P_{s+1}^{H} P_{0}+Q_{s+1}^{H} Q_{0}\right)\right\}$

$$
\begin{array}{r}
=\operatorname{Re}\left\{\operatorname { t r } \left[\left(A_{1}^{H} R_{s+1} B_{1}^{H}+\bar{A}_{2}^{H} \overline{R_{s+1}} \bar{B}_{2}^{H}\right.\right.\right. \\
+B_{3} R_{s+1}^{H} A_{3}+\overline{B_{4}} R_{s+1}^{T} \overline{A_{4}} \\
\left.+\frac{\left\|R_{s+1}\right\|^{2}}{\left\|R_{s}\right\|^{2}} P_{s}\right)^{H} P_{0} \\
+\left(C_{1}^{H} R_{s+1} D_{1}^{H}+\bar{C}_{2}^{H} \overline{R_{s+1}} \bar{D}_{2}^{H}\right. \\
+D_{3} R_{s+1}^{H} C_{3}+\overline{D_{4}} R_{k+1}^{T} \overline{C_{4}} \\
\left.\left.\left.+\frac{\left\|R_{s+1}\right\|^{2}}{\left\|R_{s}\right\|^{2}} Q_{s}\right)^{H} Q_{0}\right]\right\}
\end{array}
$$$$
=\operatorname{Re}\left\{\operatorname { t r } \left[R_{s+1}^{H} A_{1} P_{0} B_{1}+\overline{R_{s+1}} \bar{A}_{2} P_{0} \bar{B}_{2}\right.\right.
$$

$$
+R_{s+1} B_{3}^{H} P_{0} A_{3}^{H}+\overline{R_{s+1}}{\overline{B_{4}}}^{H} P_{0}{\overline{A_{4}}}^{H}
$$$$
+\frac{\left\|R_{s+1}\right\|^{2}}{\left\|R_{s}\right\|^{2}} P_{s}^{H} P_{0}+R_{s+1}^{H} C_{1} Q_{0} D_{1}
$$$$
+{\overline{R_{s+1}}}^{H} \bar{C}_{2} Q_{0} \bar{D}_{2}+R_{s+1} D_{3}^{H} Q_{0} C_{3}^{H}
$$$$
\left.\left.+\overline{R_{s+1}}{\overline{D_{4}}}^{H} Q_{0}{\overline{C_{4}}}^{H}+\frac{\left\|R_{s+1}\right\|^{2}}{\left\|R_{s}\right\|^{2}} Q_{s}^{H} Q_{0}\right]\right\}
$$$$
=\operatorname{Re}\left\{\operatorname { t r } \left[R_{s+1}^{H} A_{1} P_{0} B_{1}+\overline{\overline{R_{s+1}}} \bar{A}_{2} P_{0} \bar{B}_{2}\right.\right.
$$$$
+A_{3} P_{0}^{H} B_{3} R_{s+1}^{H}+A_{4} P_{0}^{T} B_{4} R_{s+1}^{H}
$$$$
+\frac{\left\|R_{s+1}\right\|^{2}}{\left\|R_{s}\right\|^{2}} P_{s}^{H} P_{0}+R_{s+1}^{H} C_{1} Q_{0} D_{1}
$$

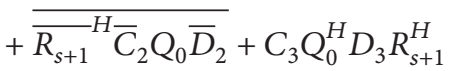$$
\left.\left.+C_{4} Q_{0}^{T} D_{4} R_{s+1}^{H}+\frac{\left\|R_{s+1}\right\|^{2}}{\left\|R_{s}\right\|^{2}} Q_{s}^{H} Q_{0}\right]\right\}
$$$$
=\operatorname{Re}\left\{\operatorname { t r } \left[R _ { s + 1 } ^ { H } \left(A_{1} P_{0} B_{1}+C_{1} Q_{0} D_{1}+A_{2} \overline{P_{0}} B_{2}\right.\right.\right.
$$

$$
\begin{gathered}
+C_{2} \overline{Q_{0}} D_{2}+A_{3} P_{0}^{H} B_{3} \\
+C_{3} Q_{0}^{H} D_{3}+A_{4} P_{0}^{T} B_{4} \\
\left.+C_{4} Q_{0}^{T} D_{4}\right) \\
\left.\left.+\frac{\left\|R_{s+1}\right\|^{2}}{\left\|R_{s}\right\|^{2}}\left(P_{s}^{H} P_{0}+Q_{s}^{H} Q_{0}\right)\right]\right\}
\end{gathered}
$$$$
=\frac{\left\|P_{0}\right\|^{2}+\left\|Q_{0}\right\|^{2}}{\left\|R_{0}\right\|^{2}} \operatorname{Re}\left\{\operatorname{tr}\left(R_{s+1}^{H}\left(R_{0}-R_{1}\right)\right)\right\}=0 \text {. }
$$

Then (26) is holds. 
From Algorithm 3 we have

$$
\begin{array}{r}
\operatorname{Re}\left\{\operatorname{tr}\left(P_{i+s+1}^{H} P_{i}+Q_{i+s+1}^{H} Q_{i}\right)\right\} \\
=\operatorname{Re}\left\{\operatorname { t r } \left[\left(A_{1}^{H} R_{i+s+1} B_{1}^{H}+\bar{A}_{2}^{H} \overline{R_{i+s+1}} \bar{B}_{2}^{H}\right.\right.\right. \\
+B_{3} R_{i+s+1}^{H} A_{3}+\overline{B_{4}} R_{i+s+1}^{T} \overline{A_{4}} \\
\left.+\frac{\left\|R_{i+s+1}\right\|^{2}}{\left\|R_{i+s}\right\|^{2}} P_{i+s}\right)^{H} P_{i} \\
+\left(C_{1}^{H} R_{i+s+1} D_{1}^{H}+\bar{C}_{2}^{H} \overline{R_{i+s+1}} \bar{D}_{2}^{H}\right. \\
+D_{3} R_{i+s+1}^{H} C_{3}+\overline{D_{4}} R_{i+s+1}^{T} \overline{C_{4}} \\
\left.\left.\left.+\frac{\left\|R_{i+s+1}\right\|^{2}}{\left\|R_{i+s}\right\|^{2}} Q_{i+s}^{H}\right)^{H}\right]\right\}
\end{array}
$$$$
=\operatorname{Re}\left\{\operatorname { t r } \left[R_{i+s+1}^{H} A_{1} P_{i} B_{1}+\overline{R_{i+s+1}} H \bar{A}_{2} P_{i} \bar{B}_{2}\right.\right.
$$$$
+R_{i+s+1} B_{3}^{H} P_{i} A_{3}^{H}+{\overline{R_{i+s+1}}}_{B_{4}}^{H} P_{i}{\overline{A_{4}}}^{H}
$$$$
+\frac{\left\|R_{i+s+1}\right\|^{2}}{\left\|R_{i+s}\right\|^{2}} P_{i+s}^{H} P_{i}+R_{i+s+1}^{H} C_{1} Q_{i} D_{1}
$$$$
+{\overline{R_{i+s+1}}}^{H} \bar{C}_{2} Q_{i} \bar{D}_{2}+R_{i+s+1} D_{3}^{H} Q_{i} C_{3}^{H}
$$$$
\left.\left.+\overline{R_{i+s+1}} \bar{D}_{4}^{H} Q_{i}{\overline{C_{4}}}^{H}+\frac{\left\|R_{i+s+1}\right\|^{2}}{\left\|R_{i+s}\right\|^{2}} Q_{i+s}^{H} Q_{i}\right]\right\}
$$$$
=\operatorname{Re}\left\{\operatorname { t r } \left[R_{i+s+1}^{H} A_{1} P_{i} B_{1}+\overline{\overline{R_{i+s+1}}} \bar{A}_{2} P_{i} \bar{B}_{2}\right.\right.
$$$$
+A_{3} P_{i}^{H} B_{3} R_{i+s+1}^{H}+A_{4} P_{i}^{T} B_{4} R_{i+s+1}^{H}
$$$$
+\frac{\left\|R_{i+s+1}\right\|^{2}}{\left\|R_{i+s}\right\|^{2}} P_{i+s}^{H} P_{i}+R_{i+s+1}^{H} C_{1} Q_{i} D_{1}
$$

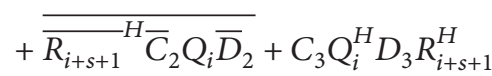$$
\left.\left.+C_{4} Q_{k}^{T} D_{4} R_{i+s+1}^{H}+\frac{\left\|R_{i+s+1}\right\|^{2}}{\left\|R_{i+s}\right\|^{2}} Q_{i+s}^{H} Q_{i}\right]\right\}
$$$$
=\operatorname{Re}\left\{\operatorname { t r } \left[R _ { i + s + 1 } ^ { H } \left(A_{1} P_{i} B_{1}+C_{1} Q_{i} D_{1}\right.\right.\right.
$$

$$
\begin{aligned}
& +A_{2} \overline{P_{i}} B_{2}+C_{2} \overline{Q_{i}} D_{2} \\
& +A_{3} P_{i}^{H} B_{3}+C_{3} P_{i}^{H} D_{3} \\
& \left.+A_{4} P_{i}^{T} B_{4}+C_{4} Q_{i}^{T} D_{4}\right)
\end{aligned}
$$$$
\left.\left.+\frac{\left\|R_{i+s+1}\right\|^{2}}{\left\|R_{i+s}\right\|^{2}}\left(P_{i+s}^{H} P_{i}+Q_{i+s}^{H} Q_{i}\right)\right]\right\}
$$

$$
\begin{aligned}
& =\frac{\left\|P_{i}\right\|^{2}+\left\|Q_{i}\right\|^{2}}{\left\|R_{i}\right\|^{2}} \operatorname{Re}\left\{\operatorname{tr}\left(R_{i+s+1}^{H}\left(R_{i}-R_{i+1}\right)\right)\right\} \\
& =\frac{\left\|P_{i}\right\|^{2}+\left\|Q_{i}\right\|^{2}}{\left\|R_{i}\right\|^{2}} \operatorname{Re}\left\{\operatorname{tr}\left(R_{i+s+1}^{H} R_{i}\right)\right\} .
\end{aligned}
$$

Also from (19) we have

$$
\begin{aligned}
& \operatorname{Re}\left\{\operatorname{tr}\left(R_{i+s+1}^{H} R_{i}\right)\right\} \\
& =\operatorname{Re}\left\{\operatorname { t r } \left[\left(R_{i+s}-\frac{\left\|R_{i+s}\right\|^{2}}{\left\|P_{i+s}\right\|^{2}+\left\|Q_{i+s}\right\|^{2}}\right.\right.\right. \\
& \times\left(A_{1} P_{i+s} B_{1}+C_{1} Q_{i+s} D_{1}\right. \\
& +A_{2} \overline{P_{i+s}} B_{2}+C_{2} \overline{Q_{i+s}} D_{2} \\
& +A_{3} P_{i+s}^{H} B_{3}+C_{3} Q_{i+s}^{H} D_{3} \\
& \left.\left.\left.\left.+A_{4} P_{i+s}^{T} B_{4}+C_{4} Q_{i+s}^{T} D_{4}\right)\right)^{H} R_{i}\right]\right\} \\
& =\operatorname{Re}\left\{\operatorname{tr}\left(R_{i+s}^{H} R_{i}\right)-\frac{\left\|R_{i+s}\right\|^{2}}{\left\|P_{i+s}\right\|^{2}+\left\|Q_{i+s}\right\|^{2}}\right. \\
& \times \operatorname{tr}\left(P_{i+s}^{H} A_{1}^{H} R_{i} B_{1}^{H}+Q_{i+s}^{H} C_{1}^{H} R_{i} D_{1}^{H}\right. \\
& +{\overline{P_{i+s}}}^{H} A_{2}^{H} R_{i} B_{2}^{H}+{\overline{Q_{i+s}}}^{H} C_{2}^{H} R_{i} D_{2}^{H} \\
& +P_{i+s} A_{3}^{H} R_{i} B_{3}^{H}+Q_{i+s} C_{3}^{H} R_{i} D_{3}^{H} \\
& \left.\left.+\overline{P_{i+s}} A_{4}^{H} R_{i} B_{4}^{H}+\overline{Q_{i+s}} C_{4}^{H} R_{i} D_{4}^{H}\right)\right\}, \\
& \operatorname{Re}\left\{\operatorname{tr}\left(R_{i+s+1}^{H} R_{i}\right)\right\} \\
& =-\frac{\left\|R_{i+s}\right\|^{2}}{\left\|P_{i+s}\right\|^{2}+\left\|Q_{i+s}\right\|^{2}} \\
& \times \operatorname{Re}\left\{\operatorname { t r } \left(P_{i+s}^{H} A_{1}^{H} R_{i} B_{1}^{H}+Q_{i+s}^{H} C_{1}^{H} R_{i} D_{1}^{H}\right.\right.
\end{aligned}
$$

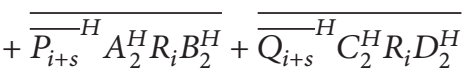

$$
\begin{aligned}
& +B_{3} R_{i}^{H} A_{3} P_{i+s}^{H}+D_{3} R_{i}^{H} C_{3} Q_{i+s}^{H} \\
& \left.\left.+\overline{B_{4}} R_{i}^{T} \overline{A_{4}} P_{i+s}^{H}+\overline{D_{4}} R_{i}^{T} \overline{C_{4}} Q_{i+s}^{H}\right)\right\}
\end{aligned}
$$




$$
\begin{gathered}
=-\frac{\left\|R_{i+s}\right\|^{2}}{\left\|P_{i+s}\right\|^{2}+\left\|Q_{i+s}\right\|^{2}} \\
\times \operatorname{Re}\left\{\operatorname { t r } \left[P _ { i + s } ^ { H } \left(A_{1}^{H} R_{i} B_{1}^{H}+\bar{A}_{2}^{H} \overline{R_{i}} \bar{B}_{2}^{H}\right.\right.\right. \\
\left.+B_{3} R_{i}^{H} A_{3}+\overline{B_{4}} R_{i}^{T} \overline{A_{4}}\right) \\
+Q_{i+s}^{H}\left(C_{1}^{H} R_{i} D_{1}^{H}+\bar{C}_{2}^{H} \bar{R}_{i} \bar{D}_{2}^{H}\right. \\
\left.\left.\left.+D_{3} R_{i}^{H} C_{3}+\overline{D_{4}} R_{i}^{T} \overline{C_{4}}\right)\right]\right\} \\
=-\frac{\left\|R_{i+s}\right\|^{2}}{\left\|P_{i+s}\right\|^{2}+\left\|Q_{i+s}\right\|^{2}} \\
\times \operatorname{Re}\left\{\operatorname { t r } \left[P_{i+s}^{H}\left(P_{i}-\frac{\left\|R_{i}\right\|^{2}}{\left\|R_{i-1}\right\|^{2}} P_{i-1}\right)\right.\right. \\
\left.\left.+Q_{i+s}^{H}\left(Q_{i}-\frac{\left\|R_{i}\right\|^{2}}{\left\|R_{i-1}\right\|^{2}} Q_{i-1}\right)\right]\right\} \\
=\frac{\left\|R_{i+s}\right\|^{2}}{\left\|P_{i+s}\right\|^{2}+\left\|Q_{i+s}\right\|^{2}} \frac{\left\|R_{i}\right\|^{2}}{\left\|R_{i-1}\right\|^{2}} \\
\times \operatorname{Re}\left\{\operatorname{tr}\left(P_{i+s}^{H} P_{i-1}+Q_{i+s}^{H} Q_{i-1}\right)\right\} .
\end{gathered}
$$

Repeating (28) and (29), one can easily obtain for certain $\alpha$ and $\beta$

$$
\begin{aligned}
\operatorname{tr}\left(P_{i+s+1}^{H} P_{i}+Q_{i+s+1}^{H} Q_{i}\right) & =\alpha\left[\operatorname{tr}\left(P_{s+1}^{H} P_{1}+Q_{s+1}^{H} Q_{1}\right)\right] \\
\operatorname{tr}\left(R_{i+s+1}^{H} R_{i}\right) & =\beta\left[\operatorname{tr}\left(R_{s+1}^{H} R_{1}\right)\right] .
\end{aligned}
$$

Combining these two relations with (26) implies that (24) holds for $l=s+1$. From Steps 1 and 2 the conclusion holds by the principle of induction. With the above two lemmas, we have the following theorem.

Theorem 6 (see [32]). If the matrix equation (1) is consistent, then a solution can be obtained within finite iteration steps by using Algorithm 3 for any initial matrices $V_{1}, W_{1}$.

\section{Numerical Example}

In this section, we present numerical example to illustrate the application of our proposed methods.

Example 7. In this example we illustrate our theoretical results of Algorithm 3 for solving the system of matrix equation:

$$
\begin{gathered}
A_{1} V B_{1}+C_{1} W D_{1}+A_{2} \bar{V} B_{2}+C_{2} \bar{W} D_{2}+A_{3} V^{H} B_{3} \\
+C_{3} W^{H} D_{3}+A_{4} V^{T} B_{4}+C_{4} W^{T} D_{4}=E .
\end{gathered}
$$

Because of the influence of the error of calculation, the residual $R(k)$ is usually unequal to zero in this process of the iteration. We regard the matrix $R(k)$ as a zero matrix if $R(k)<10^{-10}$.

Given

$$
\begin{aligned}
& A_{1}=\left[\begin{array}{ccc}
2+3 i & -i & 1+i \\
5 & 1+2 i & -3
\end{array}\right] \\
& A_{2}=\left[\begin{array}{ccc}
2+3 i & -i & 1+i \\
5 & 1+2 i & -3
\end{array}\right] \\
& A_{3}=\left[\begin{array}{ccc}
0 & 2-i & i \\
-1+3 i & 2 & 0
\end{array}\right], \quad A_{4}=\left[\begin{array}{ccc}
0 & 1-3 i & 1+i \\
0 & 4+i & -3 i
\end{array}\right] \text {, } \\
& C_{1}=\left[\begin{array}{ccc}
1+2 i & 3-i & 4 \\
-i & 2 i & -3
\end{array}\right], \quad C_{2}=\left[\begin{array}{ccc}
3+2 i & 0 & 1+i \\
0 & 4 i & 1-2 i
\end{array}\right], \\
& C_{3}=\left[\begin{array}{ccc}
1-3 i & 2 i & -3 i \\
1 & 2+3 i & 4 i
\end{array}\right], \quad C_{4}=\left[\begin{array}{ccc}
1-2 i & 0 & 2 \\
3-i & 1+i & -1
\end{array}\right], \\
& B_{1}=\left[\begin{array}{cc}
4+i & -i \\
0 & 1-i \\
4 i & 2+2 i
\end{array}\right], \quad B_{2}=\left[\begin{array}{cc}
0 & i \\
1+i & 0 \\
-1-i & 3 i
\end{array}\right] \text {, } \\
& B_{3}=\left[\begin{array}{cc}
0 & 1 \\
-3 i & 4+i \\
5 & 1+2 i
\end{array}\right], \quad B_{4}=\left[\begin{array}{cc}
3+i & -1-i \\
0 & 2-i \\
-1+i & 2
\end{array}\right] \text {, } \\
& D_{1}=\left[\begin{array}{cc}
0 & 0 \\
1-3 i & -i \\
2 i & -3 i
\end{array}\right], \quad D_{2}=\left[\begin{array}{cc}
0 & i \\
1+i & 0 \\
-1-i & 3 i
\end{array}\right] \text {, } \\
& D_{3}=\left[\begin{array}{cc}
0 & 1 \\
-3 i & 4+i \\
5 & 1+2 i
\end{array}\right], \quad D_{4}=\left[\begin{array}{cc}
3 i & -2+i \\
0 & i \\
-2 i & -4 i
\end{array}\right] \text {, } \\
& E=\left[\begin{array}{cc}
42+55 i & 115+25 i \\
-38-i & 132+44 i
\end{array}\right] \text {. }
\end{aligned}
$$

Taking $V_{1}=\left[\begin{array}{ll}0 & 0 \\ 0 & 0 \\ 0 & 0\end{array}\right]$ and $W_{1}=\left[\begin{array}{ll}0 & 0 \\ 0 & 0\end{array}\right]$ we apply Algorithm 3 to compute $V_{k}, W_{k}$.

And iterating 42 steps we get

V

$=\left[\begin{array}{ccc}0.0126+1.8415 i & 0.0827+0.6381 i & 1.1221-0.9428 i \\ -0.6903+1.0185 i & 1.8818+1.0203 i & 0.9208+0.4569 i \\ 0.5344-0.4909 i & 0.9280+0.7169 i & 0.4872-0.2734 i\end{array}\right]$,

W

$=\left[\begin{array}{ccc}0.4218-0.9710 i & 0.1763+0.5183 i & 1.1331-0.0432 i \\ 0.6273-0.1216 i & -0.3902+0.4313 i & 0.6240+0.9828 i \\ -0.7011-0.3418 i & 0.3695+1.7627 i & -0.3032+0.7073 i\end{array}\right]$

which satisfy the matrix equation:

$$
\begin{gathered}
A_{1} V B_{1}+C_{1} W D_{1}+A_{2} \bar{V} B_{2}+C_{2} \bar{W} D_{2}+A_{3} V^{H} B_{3} \\
+C_{3} W^{H} D_{3}+A_{4} V^{T} B_{4}+C_{4} W^{T} D_{4}=E .
\end{gathered}
$$




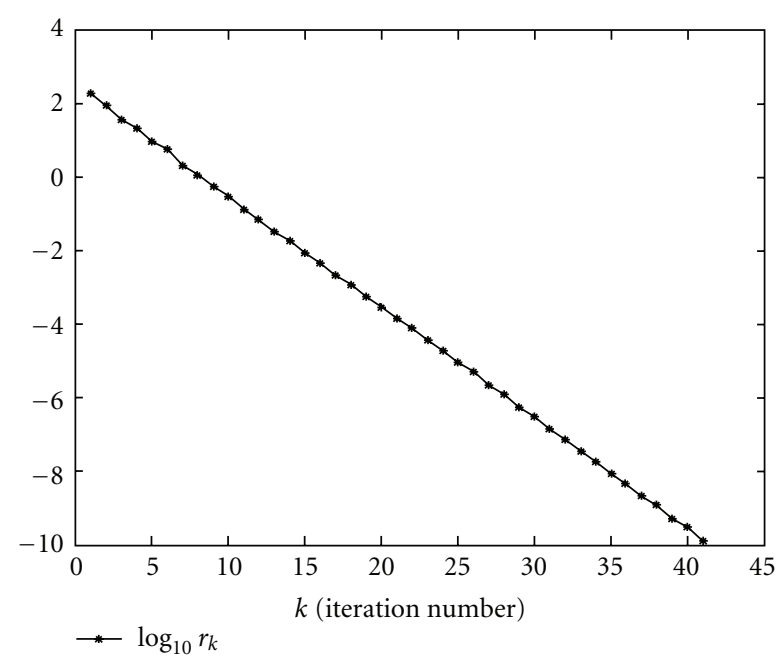

FIGURE 1: The relation between the number of iterations and residual for the example.

With the corresponding residual

$$
\begin{aligned}
&\left\|R_{42}\right\| \\
&=\| E-A_{1} V_{42} B_{1}-C_{1} W_{42} D_{1}-A_{2} \overline{V_{42}} B_{2}-C_{2} \bar{W}_{42} D_{2} \\
& \quad \quad-A_{3} V_{42}^{H} B_{3}-C_{3} W_{42}^{H} D_{3}-A_{4} V_{42}^{T} B_{4}-C_{4} W_{42}^{T} D_{4} \| \\
&= 6.6115 \times 10^{-11} .
\end{aligned}
$$

\section{Conclusions}

The above Figure 1 shows the convergence curve for the residual function $R(k)$. In this paper, an iterative algorithm constructed to solve a complex matrix equation with conjugate and transpose of two unknowns of the form: $A_{1} V B_{1}+$ $C_{1} W D_{1}+A_{2} \bar{V} B_{2}+C_{2} \bar{W} D_{2}+A_{3} V^{H} B_{3}+C_{3} W^{H} D_{3}+A_{4} V^{T} B_{4}+$ $C_{4} W^{T} D_{4}=E$ is presented. We proved that the iterative algorithms always converge to the solution for any initial matrices. We stated and proved some lemmas and theorems where the solutions are obtained. The proposed method is illustrated by numerical example where the obtained numerical results show that our technique is very neat and efficient.

\section{References}

[1] F. Ding, P. X. Liu, and J. Ding, "Iterative solutions of the generalized Sylvester matrix equations by using the hierarchical identification principle," Applied Mathematics and Computation, vol. 197, no. 1, pp. 41-50, 2008.

[2] F. Ding and T. Chen, "Gradient based iterative algorithms for solving a class of matrix equations," IEEE Transactions on Automatic Control, vol. 50, no. 8, pp. 1216-1221, 2005.

[3] F. Ding and T. Chen, "Hierarchical gradient-based identification of multivariable discrete-time systems," Automatica, vol. 41, no. 2, pp. 315-325, 2005.
[4] F. Ding and T. Chen, "Iterative least-squares solutions of coupled Sylvester matrix equations," Systems and Control Letters, vol. 54, no. 2, pp. 95-107, 2005.

[5] F. Ding and T. Chen, "On iterative solutions of general coupled matrix equations," SIAM Journal on Control and Optimization, vol. 44, no. 6, pp. 2269-2284, 2006.

[6] F. Ding and T. Chen, "Hierarchical least squares identification methods for multivariable systems," IEEE Transactions on Automatic Control, vol. 50, no. 3, pp. 397-402, 2005.

[7] F. Ding and T. Chen, "Performance analysis of multi-innovation gradient type identification methods," Automatica, vol. 43, no. 1, pp. 1-14, 2007.

[8] A. G. Wu, X. Zeng, G. R. Duan, and W. J. Wu, "Iterative solutions to the extended Sylvester-conjugate matrix equations," Applied Mathematics and Computation, vol. 217, no. 1, pp. 130-142, 2010.

[9] B. Zhou, G. R. Duan, and Z. Y. Li, "Gradient based iterative algorithm for solving coupled matrix equations," Systems and Control Letters, vol. 58, no. 5, pp. 327-333, 2009.

[10] J. H. Bevis, F. J. Hall, and R. E. Hartwing, "Consimilarity and the matrix equation $A \bar{X}-X B=C$," in Current Trends in Matrix Theory, pp. 51-64, North-Holland, New York, NY, USA, 1987.

[11] R. A. Horn and C. R. Johnson, Matrix Analysis, Cambridge University Press, Cambridge, UK, 1990.

[12] H. Liping, "Consimilarity of quaternion matrices and complex matrices," Linear Algebra and Its Applications, vol. 331, no. 1-3, pp. 21-30, 2001.

[13] T. Jiang, X. Cheng, and L. Chen, "An algebraic relation between consimilarity and similarity of complex matrices and its applications," Journal of Physics A, vol. 39, no. 29, pp. 9215-9222, 2006.

[14] J. H. Bevis, F. J. Hall, and R. E. Hartwig, "The matrix equation $A \bar{X}-X B=C$ and its special cases," SIAM Journal on Matrix Analysis and Applications, vol. 9, no. 3, pp. 348-359, 1988.

[15] A. G. Wu, G. R. Duan, and H. H. Yu, "On solutions of the matrix equations $X F-A X=C$ and $X F-A \bar{X}=C$," Applied Mathematics and Computation, vol. 183, no. 2, pp. 932-941, 2006.

[16] A. Navarra, P. L. Odell, and D. M. Young, "Representation of the general common solution to the matrix equations $A_{1} X B_{1}=C_{1}, A_{2} X B_{2}=C_{2}$ with applications," Computers and Mathematics with Applications, vol. 41, no. 7-8, pp. 929-935, 2001.

[17] J. W. Van der Woude, "On the existence of a common solution $\mathrm{X}$ to the matrix equations $A_{i} X B_{j}=C(\mathrm{i}, \mathrm{j}) \mathrm{E} \mathrm{I}$," Linear Algebra and Its Applications, vol. 375, no. 1-3, pp. 135-145, 2003.

[18] P. Bhimasankaram, "Common solutions to the linear matrix equations $A X=C, X B=D$, and $\mathrm{EXF}=\mathrm{G}$," Sankhya Series $A$, vol. 38, pp. 404-409, 1976.

[19] S. Kumar Mitra, "The matrix equations $A X=C, X B=D$," Linear Algebra and Its Applications, vol. 59, pp. 171-181, 1984.

[20] S. K. Mitra, "A pair of simultaneous linear matrix equations $A_{1} X B_{1}=C_{1}, A_{2} X B_{2}=C_{2}$ and a matrix programming problem," Linear Algebra and Its Applications, vol. 131, pp. 107123,1990

[21] M. A. Ramadan, M. A. Abdel Naby, and A. M. E. Bayoumi, "On the explicit solutions of forms of the Sylvester and the Yakubovich matrix equations," Mathematical and Computer Modelling, vol. 50, no. 9-10, pp. 1400-1408, 2009.

[22] K. W. E. Chu, "Singular value and generalized singular value decompositions and the solution of linear matrix equations," Linear Algebra and Its Applications, vol. 88-89, pp. 83-98, 1987. 
[23] Y. X. Yuan, "The optimal solution of linear matrix equation by matrix decompositions, Math," Numerica Sinica, vol. 24, pp. 165-176, 2002.

[24] A. P. Liao and Y. Lei, "Least-squares solution with the minimum-norm for the matrix equation $(A X B, G X H)=(C, D)$," Computers and Mathematics with Applications, vol. 50, no. 3-4, pp. 539-549, 2005.

[25] Y. H. Liu, "Ranks of least squares solutions of the matrix equation $A X B=C$," Computers and Mathematics with Applications, vol. 55, no. 6, pp. 1270-1278, 2008.

[26] X. Sheng and G. Chen, "A finite iterative method for solving a pair of linear matrix equations $(A X B, C X D)=(E, F)$," Applied Mathematics and Computation, vol. 189, no. 2, pp. 1350-1358, 2007.

[27] A. G. Wu, W. Liu, and G. R. Duan, "On the conjugate product of complex polynomial matrices," Mathematical and Computer Modelling, vol. 53, no. 9-10, pp. 2031-2043, 2011.

[28] A. G. Wu, G. Feng, W. Liu, and G. R. Duan, "The complete solution to the Sylvester-polynomial-conjugate matrix equations," Mathematical and Computer Modelling, vol. 53, no. 9-10, pp. 2044-2056, 2011.

[29] A. G. Wu, B. Li, Y. Zhang, and G. R. Duan, "Finite iterative solutions to coupled Sylvester-conjugate matrix equations," Applied Mathematical Modelling, vol. 35, no. 3, pp. 1065-1080, 2011.

[30] A. G. Wu, L. Lv, and G. R. Duan, "Iterative algorithms for solving a class of complex conjugate and transpose matrix equations," Applied Mathematics and Computation, vol. 217, no. 21, pp. 8343-8353, 2011.

[31] X. Zhang, Matrix Analysis and Application, Tsinghua University Press, Beijing, China, 2004.

[32] A. G. Wu, L. Lv, and M. Z. Hou, "Finite iterative algorithms for extended Sylvester-conjugate matrix equations," Mathematical and Computer Modelling, vol. 54, pp. 2363-2384, 2011. 


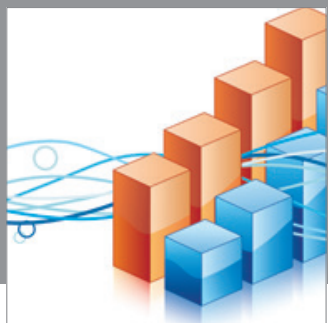

Advances in

Operations Research

mansans

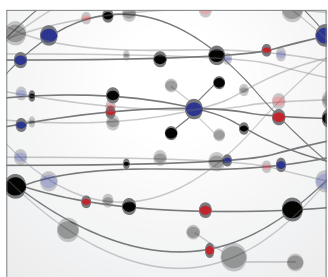

The Scientific World Journal
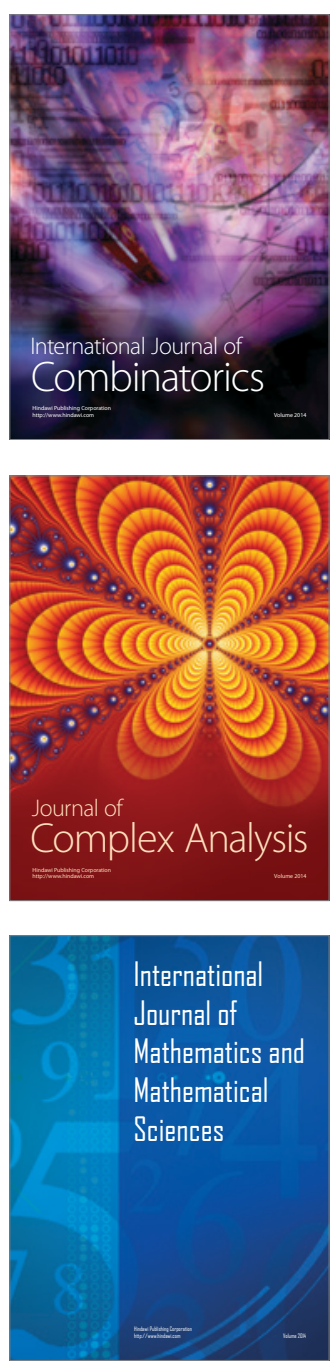
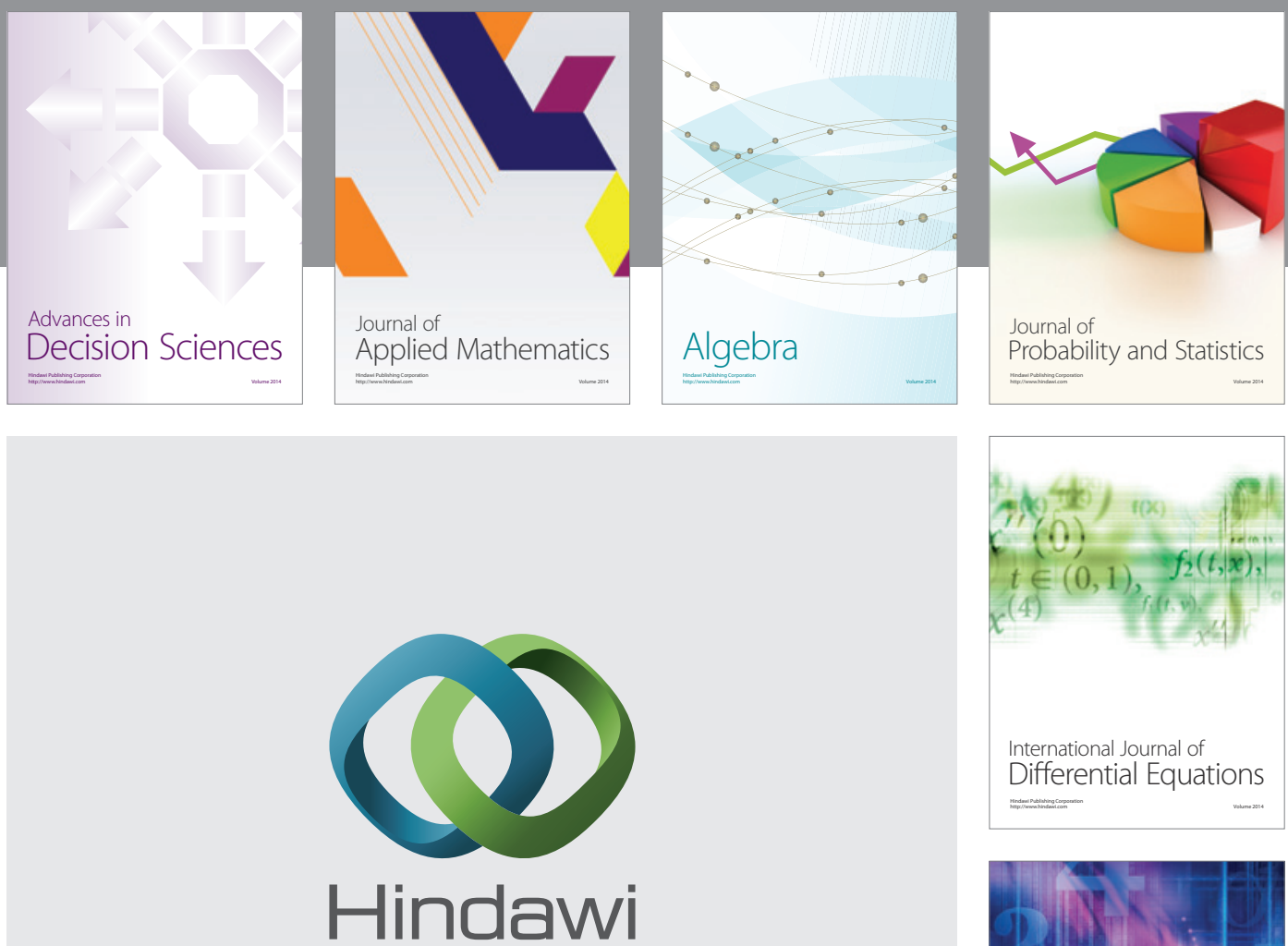

Submit your manuscripts at http://www.hindawi.com
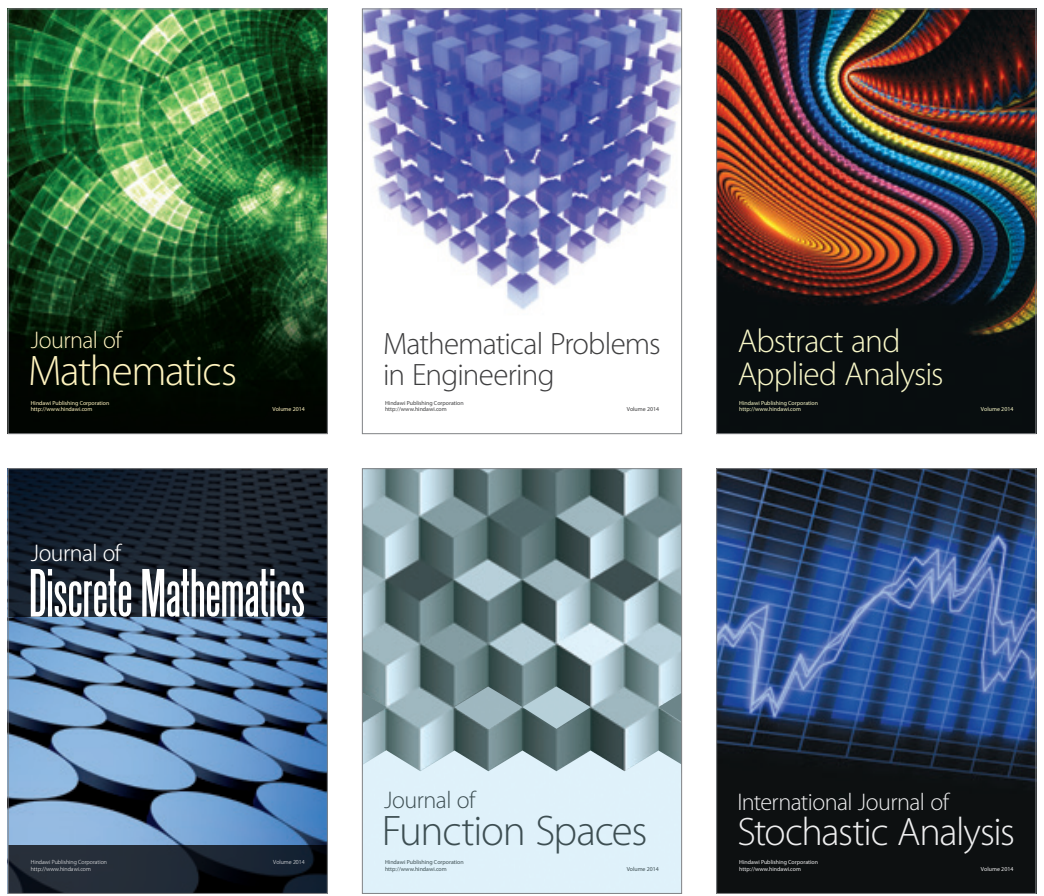

Journal of

Function Spaces

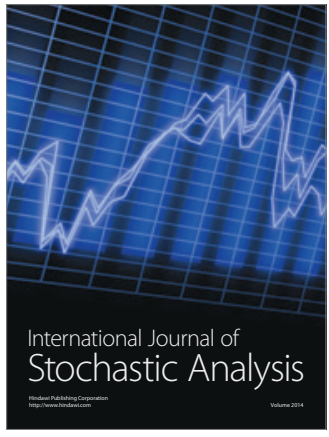

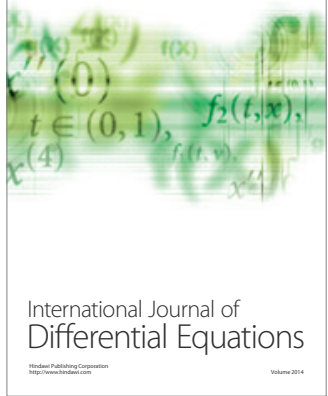
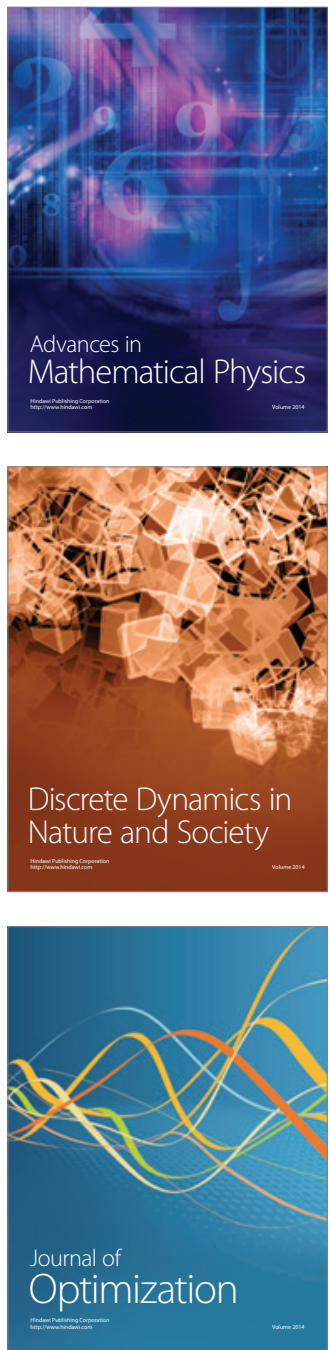